Homology, Homotopy and Applications, vol.5(1), 2003, pp.219-249

\title{
WEAK CORESTRICTION PRINCIPLE FOR NON-ABELIAN GALOIS COHOMOLOGY
}

\author{
NGUYỄ̃ QUÔĆ THǍŃG
}

(communicated by Hvedri Inassaridze)

\begin{abstract}
We introduce the notion of (Weak) Corestriction Principle and prove some relations between the validity of this principle for various connecting maps in non-abelian Galois cohomology over fields of characteristic 0 . We also prove the validity of Weak Corestriction Principle for images of coboundary maps $\mathrm{H}^{1}(k, G) \rightarrow \mathrm{H}^{2}(k, T)$, where $T$ is a finite commutative $k$-group of multiplicative type, $G$ is adjoint, semisimple and contains only almost simple factors of certain inner types.
\end{abstract}

\section{Introduction.}

Let $G$ be a commutative algebraic group defined over a field $k$ of characteristic 0 . Let $\mathrm{H}^{i}(k, G)$ denote the usual Galois cohomology $\mathrm{H}^{i}(\operatorname{Gal}(\bar{k} / k), G(\bar{k}))$. It is well-known that there exists corestriction homomorphism Cores $:=$ Cores $_{k^{\prime} / k}$ : $\mathrm{H}^{i}\left(k^{\prime}, G\right) \rightarrow \mathrm{H}^{i}(k, G)$ for any $i \geqslant 1$ and any finite extension $k^{\prime}$ of $k$, which gives rise to a map of functors $\left(G \mapsto \mathrm{H}^{i}\left(k^{\prime}, G\right)\right) \rightarrow\left(G \mapsto \mathrm{H}^{i}(k, G)\right)$. In particular, if

$$
1 \rightarrow A \stackrel{j}{\rightarrow} B \stackrel{p}{\rightarrow} C \rightarrow 1
$$

is an exact sequence of commutative algebraic $k$-groups, $\left\{\alpha_{1}, \alpha_{2}, \ldots\right\}$ (resp. $\left.\left\{\alpha_{1}^{\prime}, \alpha_{2}^{\prime}, \ldots\right\}\right)$ denotes the sequence of homomorphisms appearing in the long exact sequence of cohomology deduced from $(*)$ as cohomology of $\operatorname{Gal}(\bar{k} / k)$-modules (resp. as $\operatorname{Gal}\left(\bar{k} / k^{\prime}\right)$-modules), then we have

$$
\text { Cores } \circ \alpha_{m}^{\prime}=\alpha_{m} \circ \text { Cores }
$$

for all $m \geqslant 1$. However, if in $(*)$ one of the groups is not commutative, then it turns out that there is no corestriction map between these two long exact sequences in general. (In [R1], C. Riehm has found some sufficient conditions for the existence of corestriction map.) It leads us to the following definition. Let $A, B$ be algebraic groups defined over $k$. Assume that we are given a map of functors $f:(L \mapsto$ $\left.\mathrm{H}^{i}(L, A)\right) \rightarrow\left(L \mapsto \mathrm{H}^{j}(L, B)\right)$, where $L$ denotes a field extension of $k$, i.e., a collection of maps of cohomology sets $f_{L}: \mathrm{H}^{i}(L, A) \rightarrow \mathrm{H}^{j}(L, B)$, where $f_{L}$ is functorial in $L$.

Received March 21, 2003, revised June 30, 2003; published on July 18, 2003.

2000 Mathematics Subject Classification: Primary 11G72; Secondary 18G50, 20 G10.

Key words and phrases: Corestriction maps, Norm maps, Non-abelian Galois cohomology.

(C) 2003, Nguyêñ Quôć Thăńg. Permission to copy for private use granted. 
Assume further that among $A, B$, only $B$ (resp. $A$ ) is a commutative algebraic $k$-group and $0 \leqslant i \leqslant 1$ (resp. $0 \leqslant j \leqslant 1$ ).

Definition. We say that the Corestriction Principle over $k$ holds for the image (resp. the kernel) of $f_{k}$ if we have

$$
\operatorname{Cores}_{k^{\prime} / k}\left(\operatorname{Im}\left(f_{k^{\prime}}\right)\right) \subset \operatorname{Im}\left(f_{k}\right)
$$

(resp.

$$
\left.\operatorname{Cores}_{k^{\prime} / k}\left(\operatorname{Ker}\left(f_{k^{\prime}}\right)\right) \subset \operatorname{Ker}\left(f_{k}\right)\right)
$$

for any finite extension $k^{\prime}$ of $k$. We call a map $\mathrm{H}^{i}(k, A) \rightarrow \mathrm{H}^{j}(k, B)$ connecting if it is the usual connecting map appearing in the long exact sequence of Galois cohomology deduced from an exact sequence of $k$-groups involving $A$ and $B$. It is natural and important to investigate whether or not the Corestriction Principle always holds for connecting maps. In the case $i=1, j=2$, Rosset and Tate [RT] constructed an example showing that, in general, Corestriction Principle for the image (or kernel) does not hold. Namely, let

$$
1 \rightarrow \mu_{n} \rightarrow \mathrm{SL}_{n} \rightarrow \mathrm{PGL}_{n} \rightarrow 1
$$

be the exact sequence of algebraic $k$-groups, where $\mu_{n}$ denotes the center $(=n$-th roots of 1) of the special linear group $\mathrm{SL}_{n}$. Then they showed the following. Assume that $\mu_{n} \subset k$ and consider any finite extension $k^{\prime} / k$. Any element of the image of the connecting (boundary) map $\Delta^{\prime}: \mathrm{H}^{1}\left(k^{\prime}, \mathrm{PGL}_{n}\right) \rightarrow \mathrm{H}^{2}\left(k^{\prime}, \mu_{n}\right)$ will be called a (cohomological) symbol over $k^{\prime}$. Then via the corestriction map Cores $_{k^{\prime} / k}$, the image of a symbol is a sum (in the corresponding group) of elements from the image of $\Delta$ and may not be in the image. Thus the norm (corestriction) of a (cohomological) symbol may not be a symbol, though it is a sum of symbols. In other words, a weaker statement holds true : the corestriction of a symbol over $k^{\prime}$ lies in the group generated by the symbols over $k$. (In view of Merkurjev and Suslin's Theorem [MS], if $k$ contains a primitive $n$-th root of 1 , then we have the following stronger statement : the image of the connecting map $\Delta: \mathrm{H}^{1}\left(k, \mathrm{PGL}_{n}\right) \rightarrow \mathrm{H}^{2}\left(k, \mu_{n}\right)={ }_{n} B r(k)$ generates the whole $n$-torsion subgroup ${ }_{n} B r(k)$ of the Brauer group $B r(k)$ of $k$.)

So for a connecting map $\alpha_{k}: \mathrm{H}^{p}(k, G) \rightarrow \mathrm{H}^{q}(k, T)$, where $G, T$ are connected reductive $k$-groups, $T$ is a torus, $0 \leqslant p \leqslant 1,0 \leqslant q \leqslant 2$ and for a collection of connecting maps $\alpha_{k^{\prime}}: \mathrm{H}^{p}\left(k^{\prime}, G\right) \rightarrow \mathrm{H}^{q}\left(k^{\prime}, T\right)$ for finite extensions $k^{\prime}$ of a field $k$, it is natural to ask if the above generation phenomenon always holds.

Definition. We say that Weak Corestriction Principle holds for the image of $\alpha_{k}$, if

$$
\operatorname{Cores}_{k^{\prime} / k}\left(\operatorname{Im}\left(\alpha_{k^{\prime}}\right)\right) \subset\left\langle\operatorname{Im}\left(\alpha_{k}\right)\right\rangle,
$$

where $\langle A\rangle$ denotes the subgroup generated by $A$ in the corresponding group and Cores $_{k^{\prime} / k}$ denotes the corestriction map for the corresponding cohomology groups for the finite extension $k^{\prime} / k$.

Similarly, one may consider (Weak) Corestriction Principle for the kernel of a connecting map. Notice that some particular cases of the Corestriction Principle (in 
other terminology, Norm Principle), was proved to hold in [Gi], [Me2], [T1], [T2] under certain restrictions either on the group $G$, or on the arithmetic nature of the field $k$.

In this paper we show that the above observation made by Rosset and Tate holds in fact for a large class of groups. We would like to make a conjecture that Weak Corestriction Principle always holds over any field. Our first main result (see Theorems 2.10, 2.11) shows some interrelation between the validity of (Weak) Corestriction Principle for different kinds of connecting maps. As applicationis, one can get other counterexamples to the Corestriction Principle (e.g. for $i=j=1$ ).

As a second main objective of this paper, we investigate the Weak Corestriction Principle for images of connecting maps coming from Galois cohomology of reductive groups. Our second main result (see Theorem 4.1) is the following Weak Corestriction Principle.

Let $k$ be a field of characteristic 0 and let $\Delta: \mathrm{H}^{1}(k, G) \rightarrow \mathrm{H}^{2}(k, F)$ be the coboundary map between cohomology sets where $F$ is the center of a semisimple $k$-group $G_{1}, G=G_{1} / F$ is the adjoint $k$-group of $G_{1}$. Assume that $G$ contains only almost simple factors of classical, inner types ${ }^{1} \mathrm{~A}, \mathrm{~B}, \mathrm{C},{ }^{1} \mathrm{D}_{n}$ ( $n$ even). Let $k^{\prime}$ be a finite extension of $k$ and assume that $k$ contains $(m+1)$ th-roots of unity if $G$ contains a ${ }^{1} \mathrm{~A}_{m}$-factor. Then

$$
\operatorname{Cores}_{k^{\prime} / k}\left(\operatorname{Im}\left(\Delta \otimes k^{\prime}\right)\right) \subset\langle\operatorname{Im}(\Delta)\rangle .
$$

After some preliminary results in Section 1, in Section 2 we prove some equivalent conditions (in the form of reduction theorems), which show how different statements about (Weak) Corestriction Principle are related and how useful they are in reducing the problem to a simpler one. In Section 3 we reduce the problem to the quasi-split case. In Section 4 we prove the second main result mentioned above.

\section{Preliminary results}

In this section we present some necessary facts related with the well-known crossed-diagram construction given by Ono (which was also the notion of $z$-extensions used by Langlands), and we make some preliminary reductions. We will need the following lemmas.

Lemma 1.1. Assume that we have the following commutative diagram

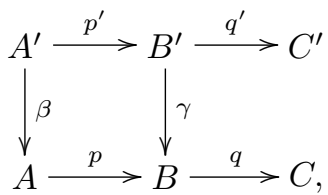

where $A, B, A^{\prime}, B^{\prime}$ are groups, the left diagram is a commutative diagram of groups. Let $e^{\prime}=q^{\prime}(1), e=q(1)$, where 1 is the identity element of the corresponding groups. Then if $\gamma\left(q^{\prime-1}\left(e^{\prime}\right)\right) \subset q^{-1}(e)$ then

$$
\beta\left(r^{\prime-1}\left(e^{\prime}\right)\right) \subset r^{-1}(e),
$$


with $r^{\prime}=q^{\prime} p^{\prime}, r=q p$.

The proof is easy so we omit it.

Recall that a connected reductive $k$-group $H$ is called (after Langglands) a $z$ extension of a $k$-group $G$ if $H$ is an extension of $G$ by an induced $k$-torus $Z$, such that the derived subgroup (called also the semisimple part) $[H, H]$ of $H$ is simply connected. For a field extension $K / k$ and an element $x \in \mathrm{H}^{1}(K, G)$, a $z$-extension $H$ of $G$ over $k$ is called $x$-lifting if $x \in \operatorname{Im}\left(\mathrm{H}^{1}(K, H) \rightarrow \mathrm{H}^{1}(K, G)\right)$.

Lemma 1.2 ([T2]). Let $G$ be a connected reductive $k$-group, $K$ a finite extension of $k, x$ an element of $\mathrm{H}^{1}(K, G)$. Then there is a z-extension

$$
1 \rightarrow Z \rightarrow H \rightarrow G \rightarrow 1
$$

of $G$, where all groups and morphisms are defined over $k$, which is $x$-lifting.

Lemma 1.3 ([T2]). Let $\alpha: G_{1} \rightarrow G_{2}$ be a homomorphism of connected reductive groups, all defined over $k, x \in \mathrm{H}^{1}\left(K, G_{1}\right)$, where $K$ is a finite extension of $k$. Then there exists a x-lifting z-extension $\alpha^{\prime}: H_{1} \rightarrow H_{2}$ of $\alpha$, i.e., $H_{i}$ is a z-extension of $G_{i}(i=1,2)$, and we have the following commutative diagram

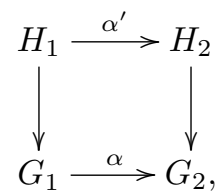

with all groups and morphisms defined over $k$.

We need the following extension of a lemma of Borovoi ([Bor], p. 45).

Lemma 1.4. Let

$$
1 \rightarrow G_{1} \rightarrow G_{2} \rightarrow G_{3} \rightarrow 1
$$

be an exact sequence of connected reductive groups over a field $k$ of characteristic 0 , $k^{\prime}$ a finite extension of $k$ and $x \in \mathrm{H}^{1}\left(k^{\prime}, G_{3}\right)$. Then there exists a z-extension of this sequence, which is $x$-lifting, i.e., an exact sequence of connected reductive $k$-groups

$$
1 \rightarrow H_{1} \rightarrow H_{2} \rightarrow H_{3} \rightarrow 1
$$

such that each $H_{i}$ is a z-extension of $G_{i}, H_{3}$ is x-lifting and the following diagram commutes

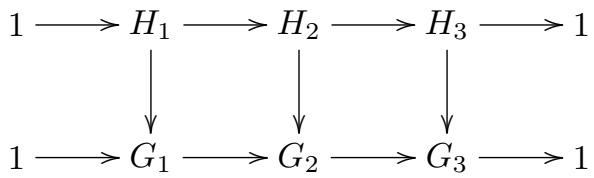


Proof. By Lemma 1.2 there exists a $z$-extension $H_{3}$ of $G_{3}$ defined over $k$, which is $x$-lifting. Now we apply the same proof of Lemma 3.10.1 of [Bor] to get the result.

Lemma 1.5. Let $A$ (resp. $\left.A^{\prime}\right)$ be a pointed set, $B$ (resp. $\left.B^{\prime}\right)$ and $C$ (resp. $\left.C^{\prime}\right)$ be groups with homomorphisms $N_{B}: B^{\prime} \rightarrow B, N_{C}: C^{\prime} \rightarrow C$. Let $f: A \rightarrow B$ and $h: A \rightarrow C$ (resp. $f^{\prime}: A^{\prime} \rightarrow B^{\prime}$ and $h^{\prime}: A^{\prime} \rightarrow C^{\prime}$ ) be maps of pointed sets and $g: B \rightarrow C, g^{\prime}: B^{\prime} \rightarrow C^{\prime}$ be homomorphisms such that $h=g \circ f, h^{\prime}=g^{\prime} \circ f^{\prime}, N_{C} \circ g^{\prime}=$ $g \circ N_{B}$. If

$$
N_{B}\left(\operatorname{Im} f^{\prime}\right) \subset\langle\operatorname{Im} f\rangle,
$$

then

$$
N_{C}\left(\operatorname{Im} h^{\prime}\right) \subset\langle\operatorname{Im} h\rangle .
$$

The proof is easy and is omitted.

Next we consider a reduction theorem for (Weak) Corestriction Principle for nonabelian Galois cohomology. Namely we show that it is possible to reduce the problem of proving the Corestriction Principle (CP) (resp. Weak Corestriction Principle (WCP)) for connecting maps related with an exact sequence of connected linear algebraic groups to the same problem where only connected reductive groups are involved. We refer the readers to [Bo] for basic notions of algebraic groups. For a linear algebraic group $G$ we denote by $G^{\circ}$ its identity component.

Theorem 1.6. Let $\alpha: \mathrm{H}^{p}(k, G) \rightarrow \mathrm{H}^{q}(k, T)$ be a connecting map of Galois cohomology, which is induced from an exact sequence of connected linear algebraic groups

$$
1 \rightarrow A \rightarrow B \rightarrow C \rightarrow 1,
$$

where all of them are defined over $k$ and $T$ is commutative. Then there is a connecting map $\alpha_{1}: \mathrm{H}^{p}\left(k, G_{1}\right) \rightarrow \mathrm{H}^{q}\left(k, T_{1}\right)$, with $T_{1}$ commutative, induced from an exact sequence of connected reductive k-groups $1 \rightarrow A_{1} \rightarrow B_{1} \rightarrow C_{1} \rightarrow 1$ canonically attached to $(*)$, such that if the CP (resp. WCP) holds for the image of $\alpha_{1}$ then the same holds for $\alpha$. The same is true for the connecting map $\mathrm{H}^{1}(k, G) \rightarrow \mathrm{H}^{1}(k, T)$, induced from a $k$-morphism of connected $k$-groups $f: G \rightarrow T$, where $T$ is commutative.

Proof. The proof consists of a case-by-case consideration, depending on the values of $p, q$.

Case $p=q=0$. Since $p=q$, we are given an exact sequence of linear connected algebraic groups

$$
1 \rightarrow G_{1} \rightarrow G \stackrel{\pi}{\rightarrow} T \rightarrow 1
$$

Here $G_{1}$ is considered as a subgroup of $G$. We have $T=T_{s} \times T_{u}$, where $T_{s}$ is the maximal torus of $T$ and $T_{u}$ is the unipotent part of $T$, all are defined over $k$. Let $G=L \cdot U$ be a Levi decomposition of $G$, where $U=R_{u}(G)$ the unipotent radical of $G$ and $L$ is a maximal connected reductive $k$-subgroup of $G$. Since the image of $U$ (resp. of $L$ ) via $\pi$ is a unipotent (resp. reductive) group, it follows that $\pi(L)=T_{s}$, 
$\pi(U)=T_{u}$. Let $k^{\prime} / k$ be any finite extension of $k, g^{\prime} \in G\left(k^{\prime}\right)$. Since $G=L U$ is a semidirect product we have $G\left(k^{\prime}\right)=L\left(k^{\prime}\right) U\left(k^{\prime}\right)$, so $g^{\prime}=l^{\prime} u^{\prime}, l^{\prime} \in L\left(k^{\prime}\right), u^{\prime} \in U\left(k^{\prime}\right)$. Let $N=N_{k^{\prime} / k}: T\left(k^{\prime}\right) \rightarrow T(k)$ be the norm map. Then $N$ induces the norm maps, denoted also by $N, N: T_{s}\left(k^{\prime}\right) \rightarrow T_{s}(k)$, and $N: T_{u}\left(k^{\prime}\right) \rightarrow T_{u}(k)$. Let $\pi\left(u^{\prime}\right)=v^{\prime} \in T_{u}\left(k^{\prime}\right), N\left(v^{\prime}\right)=v \in T_{u}(k)$. Denote by $K_{u}$ the kernel of the restriction of $\pi$ to $U$. Then $K_{u}$ is a connected unipotent normal $k$-subgroup of $U$. It is well-known that the first Galois cohomology of unipotent $k$-groups over perfect fields is trivial (see [Se], Chap. III), so from this it follows that $\pi\left(U\left(k^{\prime}\right)\right)=T_{u}\left(k^{\prime}\right), \pi(U(k))=T_{u}(k)$. So we have

$$
\begin{aligned}
N\left(\pi\left(g^{\prime}\right)\right) & =N\left(\pi\left(l^{\prime} u^{\prime}\right)\right) \\
& =N\left(\pi\left(l^{\prime}\right) \pi\left(u^{\prime}\right)\right) \\
& =N\left(\pi\left(l^{\prime}\right)\right) N\left(\pi\left(u^{\prime}\right)\right) \\
& =N\left(\pi\left(l^{\prime}\right)\right) \pi(u)
\end{aligned}
$$

where $u \in U(k)$. Therefore to prove that $N\left(\pi\left(g^{\prime}\right)\right) \in \pi(G(k))$ is equivalent to proving that $N\left(\pi\left(l^{\prime}\right)\right) \in \pi(G(k))$. If $N\left(\pi\left(l^{\prime}\right)\right)=\pi(l v)$, with $l \in L(k), v \in U(k)$, then $\pi(v)=1$, since $N\left(\pi\left(l^{\prime}\right)\right) \in T_{s}(k)$, so $N\left(\pi\left(l^{\prime}\right)\right)=\pi(l)$. Thus to prove that $N\left(\pi\left(G\left(k^{\prime}\right)\right)\right) \subset \pi(G(k))$ is equivalent to proving that $N\left(\pi\left(L\left(k^{\prime}\right)\right)\right) \subset \pi(L(k))$. Now we consider the following commutative diagram

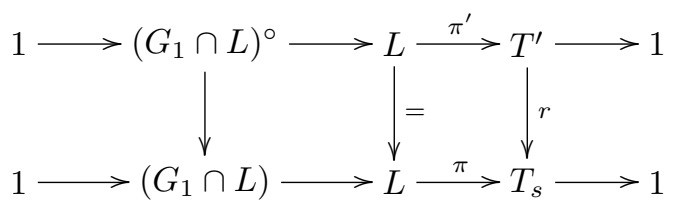

Here $T^{\prime}=L /\left(G_{1} \cap L\right)^{\circ}$ is a torus. For $x \in L$, we have $x\left(G_{1} \cap L\right)^{\circ} x^{-1}=\left(G_{1} \cap L\right)^{\circ}$, since $G_{1} \cap L$ is a normal subgroup of $L$. Furthermore, if $V$ is the unipotent radical of $\left(G_{1} \cap L\right)^{\circ}$ then $x V x^{-1}=V$ as it is not hard to see. Thus $V \subset R_{u}(L)=\{1\}$, and $\left(G_{1} \cap L\right)^{\circ}$ is a connected reductive subgroup of $L$. Now the decomposition $\pi=r \circ \pi^{\prime}$ shows that the (CP) for the image of $\pi$ follows from that for $\pi^{\prime}$, hence one may pass further to the case where all groups involved are connected and reductive.

Case $p=0, q=1$. We are given an exact sequence $1 \rightarrow T \rightarrow G_{1} \rightarrow G \rightarrow 1$ of connected groups with $T$ commutative. Let $T=T_{s} \times T_{u}$ be the decomposition of $T$ into semisimple and unipotent parts. We have the following commutative diagram

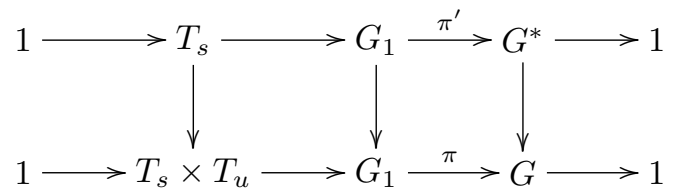

where $T$ is embedded into $G_{1}, G^{*}=G_{1} / T_{s}$. Since $T$ is normal in $G_{1}$, as in the case $p=q=0$ we see that $T_{u}$ is also normal in $G_{1}$, so $G \simeq G^{*} / T_{u}\left(T_{u}\right.$ is embeded into 
$\left.G^{*}\right)$. Since the cohomology of $T_{u}$ is trivial, this case is clear.

Case $p=q=1$. We are given an exact sequence $1 \rightarrow G_{1} \rightarrow G \stackrel{\pi}{\rightarrow} T \rightarrow 1$, with $T$ commutative and all of them are connected linear algebraic $k$-groups. As in the case $p=q=0$, we let $T=T_{s} \times T_{u}, T_{s}$ is the maximal torus of $T, T_{u}$ is the unipotent part of $T, G=L U$ is a Levi decomposition of $G$. So $\pi(L)=T_{s}, \pi(U)=T_{u}$. If $S$ denotes the connected center of $L$, then $L=S[L, L]$, thus we have $\pi(S)=T_{s}$. Let $g \in \mathrm{H}^{1}\left(k^{\prime}, G\right), t=\pi^{*}(g) \in \mathrm{H}^{1}\left(k^{\prime}, T\right)$. We know that since the 1-Galois cohomology of unipotent groups are trivial, there is canonical bijections $\mathrm{H}^{1}\left(k^{\prime}, G\right) \simeq \mathrm{H}^{1}\left(k^{\prime}, L\right)$, $\mathrm{H}^{1}\left(k^{\prime}, T\right) \simeq \mathrm{H}^{1}\left(k^{\prime}, T_{s}\right)$. Hence we may use the same commutative diagram as in the case $p=q=0$ to reduce our problem of proving the CP (resp. WCP) for the image of the connecting map $\mathrm{H}^{1}(k, G) \rightarrow \mathrm{H}^{1}(k, T)$ to that of the connecting map $\mathrm{H}^{1}(k, L) \rightarrow \mathrm{H}^{1}\left(k, T_{s}\right)$. Then, with the same notation used there, it suffices to prove the $\mathrm{CP}$ (resp. WCP) for the image of the connecting map $\mathrm{H}^{1}(k, L) \rightarrow \mathrm{H}^{1}\left(k, T^{\prime}\right)$, where $\operatorname{Ker}\left(L \rightarrow T^{\prime}\right)$ is now a connected reductive $k$-group, and we are done.

Case $p=1, q=2$. We use the same notation as in the case $p=0, q=1$. We are given an exact sequence $1 \rightarrow T \rightarrow G_{1} \rightarrow G \rightarrow 1$ with $T$ commutative. We arrive again at the following commutative diagram as in the case $p=0, q=1$ :

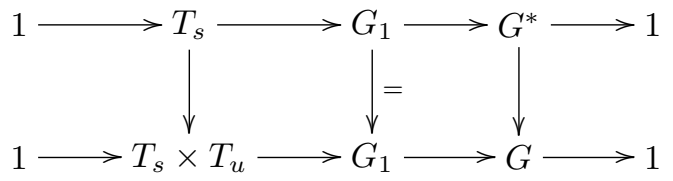

Since $T_{u}$ is commutative, we have $\mathrm{H}^{2}\left(k, T_{u}\right)=0$ by a theorem of Serre ([Se], Chap. III), so we have canonical isomorphism $\mathrm{H}^{2}\left(k, T_{s} \times T_{u}\right) \simeq \mathrm{H}^{2}\left(k, T_{s}\right)$. Hence $G^{*} / T_{u} \simeq G$ (here $T_{u}$ is embedded into $G^{*}$ ) and we have the following exact sequence for any extension $l / k$

$$
\mathrm{H}^{1}\left(l, G^{*}\right) \stackrel{p}{\rightarrow} \mathrm{H}^{1}(l, G) \rightarrow \mathrm{H}^{2}\left(l, T_{u}\right)=(0),
$$

and the following commutative diagram

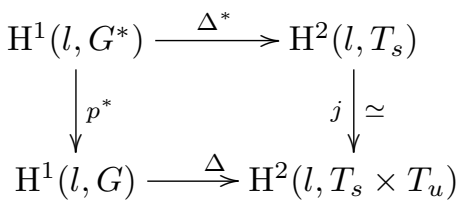

We have a similar diagram where $l$ is replaced by $k^{\prime}$ and $k$, where $k^{\prime}$ is a finite extension of $k$. Since $p^{*}$ is surjective for any extension $l / k$, it is clear that to prove $\mathrm{CP}$ for the image of $\Delta$ it suffices to prove the same thing for the image of $\Delta^{*}$. We claim that the same is true for WCP. Indeed, let $k^{\prime} / k$ be a finite extension, $g^{\prime} \in \mathrm{H}^{1}\left(k^{\prime}, G\right)$. Let $g^{*} \in \mathrm{H}^{1}\left(k^{\prime}, G^{*}\right)$ such that $p^{*}\left(g^{*}\right)=g^{\prime}$. Assuming the WCP for $\Delta^{*}$, we have

$$
\operatorname{Cores}_{k^{\prime} / k}\left(\Delta^{*}\left(g^{*}\right)\right)=\sum_{i} \Delta^{*}\left(g_{i}^{*}\right),
$$


where $g_{i}^{*} \in \mathrm{H}^{1}\left(k, G^{*}\right)$ for all $i$. Due to the functoriality we have

$$
\begin{aligned}
\operatorname{Cores}_{k^{\prime} / k}\left(\Delta\left(g^{\prime}\right)\right) & =\operatorname{Cores}_{k^{\prime} / k}\left(\Delta p^{*}\left(g^{*}\right)\right) \\
& =\operatorname{Cores}_{k^{\prime} / k}\left(j\left(\Delta^{*}\left(g^{*}\right)\right)\right. \\
& =j\left(\operatorname{Cores}_{k^{\prime} / k}\left(\Delta^{*}\left(g^{*}\right)\right)\right) \\
& =j\left(\sum_{i} \Delta^{*}\left(g_{i}^{*}\right)\right) \\
& =\sum_{i} j\left(\Delta^{*}\left(g_{i}^{*}\right)\right) \\
& =\sum_{i} \Delta p^{*}\left(g_{i}^{*}\right) \\
& =\sum_{i} \Delta\left(g_{i}\right)
\end{aligned}
$$

with $g_{i}=p^{*}\left(g_{i}^{*}\right)$ as required.

Next we consider the validity of CP (resp. WCP) for the kernel of a connecting map. In a similar way we have the following result.

Theorem 1.7. Let $\alpha: \mathrm{H}^{p}(k, T) \rightarrow \mathrm{H}^{q}(k, G)$ be a connecting map of Galois cohomology, which is induced from an exact sequence of connected linear algebraic groups

$$
1 \rightarrow A \rightarrow B \rightarrow C \rightarrow 1
$$

where all of them are defined over $k$ and $T$ is commutative. Then there is a connecting map $\alpha_{1}: \mathrm{H}^{p}\left(k, T_{1}\right) \rightarrow \mathrm{H}^{q}\left(k, G_{1}\right)$, with $T_{1}$ commutative, induced from an exact sequence of connected reductive k-groups $1 \rightarrow A_{1} \rightarrow B_{1} \rightarrow C_{1} \rightarrow 1$ canonically attached to $(*)$, such that if the CP (resp. WCP) holds for the kernel of $\alpha_{1}$ then the same holds for $\alpha$. The same is true for the connecting map $\mathrm{H}^{1}(k, T) \rightarrow \mathrm{H}^{1}(k, G)$, induced from a k-morphism of connected $k$-groups $f: T \rightarrow G$, where $T$ is commutative.

Proof. Since $\alpha$ is a connecting map in the long exact sequence of Galois cohomology of algebraic groups, there are two possibilities : either $\operatorname{Ker}(\alpha)=\operatorname{Im}(\beta)$ for some other connecting map $\beta$, or there is no such a $\beta$. In the first case we are reduced to Theorem 1.1, hence we need only consider the second case. Then we have $p=q$.

Case $p=q=0$. We are given a $k$-morphism of algebraic $k$-groups $T \stackrel{f}{\rightarrow} G$, where $T$ is commutative. Let $S_{1}=\operatorname{Ker}(f), S_{2}=\operatorname{Im}(f)$, so we have the following exact sequence $1 \rightarrow S_{1} \rightarrow T \rightarrow S_{2} \rightarrow 1$, and this case becomes trivial.

Case $p=q=1$. The above morphism $f$ induces the connecting map $f^{*}: \mathrm{H}^{1}(k, T) \rightarrow$ $\mathrm{H}^{1}(k, G)$. Notice that it induces also the following sequence of groups

$$
1 \rightarrow S_{1} \rightarrow T \stackrel{p}{\rightarrow} S_{2} \stackrel{i}{\rightarrow} G,
$$

and $f=i \circ p$. Now the assertion follows from Lemma 1.1. 
From Theorems 1.6 and 1.7 it follows that in the study of (weak) corestriction principle we may restrict ourselves to the case of connected reductive groups.

\section{2. (Weak) Corestriction Principle : equivalent relations}

In this section we will discuss some relations between the validity of (Weak) Corestriction Principles for connecting maps of various types. For simplicity we consider only connected reductive groups. If $G$ is a connected reductive $k$-group, there exist canonical maps $a b_{G}^{i}: \mathrm{H}^{i}(k, G) \rightarrow \mathrm{H}_{a b}^{i}(k, G)(i=0,1)$. Here $\mathrm{H}_{a b}^{*}$ denotes the abelianized Galois cohomology in the sense of Borovoi - Kottwitz theory and $a b_{G}^{*}$ denotes the canonical map between the cohomologies. We refer the reader to [Bor] for basic notions and properties of abelianized Galois cohomology of linear algebraic groups.

2.0. Let $k$ be a field of characteristic 0 and for a connected reductive $k$-group $G$, we use the following notation. Denote by $\tilde{G}$ (resp. $\bar{G}$ ) the simply connected covering (resp. the adjoint) group of the semisimple part $G^{\prime}:=[G, G]$ of $G$, and denote by $\tilde{F}=\operatorname{Ker}(\tilde{G} \rightarrow \bar{G}), F^{\prime}=\operatorname{Ker}\left(G^{\prime} \rightarrow \bar{G}\right)$ the corresponding kernels. We consider the following statements.

a) The (Weak) Corestriction Principle holds for the image of any connecting map $\alpha: \mathrm{H}^{p}(k, G) \rightarrow \mathrm{H}^{q}(k, T)$, where $G, T$ are connected reductive $k$-groups, with $T$ a torus and with given $p, q$ satisfying $0 \leqslant p \leqslant 1,0 \leqslant p \leqslant q \leqslant p+1$.

b) The (Weak) Corestriction Principle holds for the image of the functorial map $a b_{G}^{p}: \mathrm{H}^{p}(k, G) \rightarrow \mathrm{H}_{a b}^{p}(k, G)$, for any connected reductive $k$-group $G$ and given $p$, $0 \leqslant p \leqslant 1$.

c) The (Weak) Corestriction Principle holds for the image of the coboundary map $\mathrm{H}^{p}(k, G) \rightarrow \mathrm{H}^{p+1}(k, T)$, for any exact sequence $1 \rightarrow T \rightarrow G_{1} \rightarrow G \rightarrow 1$ of reductive $k$-groups, where $G_{1}$ is connected, $G$ is semisimple, $T$ is a central subgroup, and $p$ is given, $0 \leqslant p \leqslant 1$.

d) The same statement as in $c$ ), but $G_{1}$ and $G$ are supposed to be semisimple groups.

e) The (Weak) Corestriction Principle holds for the image of the coboundary map $\mathrm{H}^{p}(k, \bar{G}) \rightarrow \mathrm{H}^{p+1}\left(k, F^{\prime}\right)$, for any exact sequence $1 \rightarrow F^{\prime} \rightarrow G_{1} \rightarrow \bar{G} \rightarrow 1$ of reductive $k$-groups, where $G_{1}$ is semisimple, $\bar{G}$ is adjoint, $F^{\prime}$ is a finite central subgroup and $p$ is given, $0 \leqslant p \leqslant 1$.

f) The (Weak) Corestriction Principle holds for the image of coboundary map $\mathrm{H}^{p}(k, \bar{G}) \rightarrow \mathrm{H}^{p+1}(k, \tilde{F})$, for any adjoint group $\bar{G}$ with fundamental group $\tilde{F}$ and for given $p, 0 \leqslant p \leqslant 1$. 
For the statements $a)$ - $f$ ) considered above, let us denote by $x(p, q)($ resp. $y(p))$ the statement $x$ ) (resp. $y)$ ) evaluated at $(p, q)$, for $0 \leqslant p \leqslant q \leqslant 2$. For example, $a(1,2)$ means the statement $a$ ) with $p=1, q=2$, or $f(1)$ means the statement $f$ ) with $p=1$. We say that the statement $x$ ) holds if for any possible values of $(p, q)$, the corresponding statement is true. Note that for any $p, 0 \leqslant p \leqslant 1$, we have obvious imlications : $c(p) \Rightarrow d(p) \Rightarrow e(p) \Rightarrow f(p)$, i.e., $c) \Rightarrow d) \Rightarrow e) \Rightarrow f)$.

The relations between these statements are given in the following results. We will give the proof only in the case of Weak Corestriction Principle since all proofs hold true simultaneously for Corestriction and Weak Corestriction Principles, except possibly Proposition 2.9. (There, in the part $b$ ), we have to restrict ourselves to Corestriction Principle only.)

Proposition 2.1. For a given connected reductive $k$-group $G$, and a given $p$ with $0 \leqslant p \leqslant 1$, if the (Weak) Corestriction Principle holds for the image of ab $b_{G}^{p}$ then it also holds for any connecting map (if any) $\mathrm{H}^{p}(k, G) \rightarrow \mathrm{H}^{q}(k, T)$ where $T$ is a $k$-torus and $0 \leqslant q \leqslant 2$. In particular, if $b(p)$ holds for any $p$ then $a(p, q)$ holds for any pairs $(p, q)$ which make sense.

Proof. The proof follows immediately from the functoriality of the maps $a b_{G}^{p}$ : $\mathrm{H}^{p}(k, G) \rightarrow \mathrm{H}_{a b}^{p}(k, G), p=0,1$, proved in [Bor].

Proposition 2.2. For a given connected reductive group $G$, with a $z$-extension $H$, if the (Weak) Corestriction Principle holds for $a b_{H}^{p}$ for some $p(0 \leqslant p \leqslant 1)$ then the same holds for $G$. In particular, if $b(p)$ holds for connected reductive k-groups with simply connected semisimple parts then $b(p)$ holds itself.

Proof. For any finite extension $k^{\prime}$ of $k$ let $\theta \in \mathrm{H}^{p}\left(k^{\prime}, G\right)$ be any element. We choose a $\theta$-lifting $z$-extension, all defined over $k: 1 \rightarrow Z \rightarrow H \rightarrow G \rightarrow 1$, which is possible due to Lemma 1.2. Recall that $H$ is a connected reductive $k$-group with simply connected semisimple part and $Z$ is an induced $k$-torus. Let denote the induced (connecting) maps

$$
\begin{gathered}
\phi: \mathrm{H}^{p}(k, H) \rightarrow \mathrm{H}^{p}(k, G), \\
\psi: \mathrm{H}^{p}\left(k^{\prime}, H\right) \rightarrow \mathrm{H}^{p}\left(k^{\prime}, G\right),
\end{gathered}
$$

and let $\phi^{\prime}$ and $\psi^{\prime}$ stand for similar maps where $\mathrm{H}^{p}$ is replaced by $\mathrm{H}_{a b}^{p}$.

We have the following commutative diagram, where two skew (south - east) arrows are corestriction maps for abelian Galois cohomology. Here all the vertical maps are the maps $a b^{\prime}$, where $a b^{\prime}$ will denote the same map when we restrict to $k^{\prime}$ : 


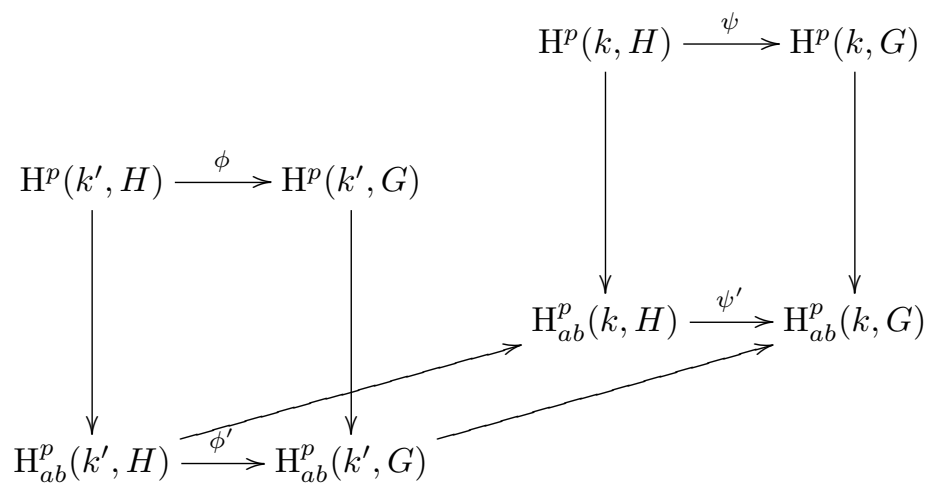

Let $\eta \in \mathrm{H}^{p}\left(k^{\prime}, H\right)$ such that $\phi(\eta)=\theta$. Then

$$
a b_{G}^{\prime}(\theta)=a b_{G}^{\prime}(\phi(\eta))=\phi^{\prime}\left(a b_{H}^{\prime}(\eta)\right) .
$$

Assuming that $d$ ) holds for $H$, then there are $\alpha_{i} \in \mathrm{H}^{p}(k, H)$ such that

$$
\sum_{i} a b_{H}\left(\alpha_{i}\right)=\operatorname{Cores}\left(a b_{H}^{\prime}(\eta)\right) \text {. }
$$

Hence

$$
\begin{aligned}
\operatorname{Cores}\left(a b_{G}^{\prime}(\theta)\right) & =\operatorname{Cores}\left(a b_{G}^{\prime}(\phi(\eta))\right) \\
& =\operatorname{Cores}\left(\phi^{\prime}\left(a b_{H}^{\prime}(\eta)\right)\right) \\
& =\psi^{\prime}\left(\operatorname{Cores}\left(a b_{H}^{\prime}(\eta)\right)\right) \\
& =\psi^{\prime}\left(\sum_{i} a b_{H}\left(\alpha_{i}\right)\right) \\
& =\sum_{i} a b_{G}\left(\psi\left(\alpha_{i}\right)\right)
\end{aligned}
$$

as required.

Proposition 2.3. Assume that for any $k$-morphism $G \rightarrow T$ of connected reductive $k$-groups, with $T$ a torus, the (Weak) Corestriction Principle holds for the image of the induced connecting map $\mathrm{H}^{p}(k, G) \rightarrow \mathrm{H}^{p}(k, T)$, and for some $p, 0 \leqslant p \leqslant 1$. Then the same holds for $a b_{G}^{p}: \mathrm{H}^{p}(k, G) \rightarrow \mathrm{H}_{a b}^{p}(k, G)$, i.e., $a(p, p) \Rightarrow b(p)$. In particular, if a) holds, then b) holds.

Proof. By Proposition 2.2, we may assume that $G^{\prime}$ is simply connected. By [Bor] we have

$$
\mathrm{H}_{a b}^{p}(k, G)=\mathrm{H}^{p}\left(k, G / G^{\prime}\right),
$$

hence $a b_{G}^{p}$ becomes just connecting map $(p=0,1)$. Since $G / G^{\prime}$ is a torus, the proposition follows. 
Proposition 2.4. If the statement a) holds for $p=q=0$ (resp. $p=q=1)$ then it also holds for $p=0, q=1$ (resp. $p=1, q=2)$, i.e., we have $a(0,0) \Rightarrow a(0,1), a(1,1) \Rightarrow$ $a(1,2)$.

Proof. It follows from the equivalence $a) \Leftrightarrow d$ ) above, Proposition 2.2, from the functoriality of the map $a b_{G}: \mathrm{H}^{p}(k, G) \rightarrow \mathrm{H}_{a b}^{p}(k, G)$ and the fact that for a connected reductive $k$-group $H$ with $H^{\prime}:=[H, H]$ simply connected, $\mathrm{H}_{a b}^{p}(k, H)=\mathrm{H}^{p}\left(k, H / H^{\prime}\right)$ (by definition) (see [Bor]).

Proposition 2.5. Let $\bar{G}$ be an adjoint semisimple k-group with fundamental group $\tilde{F}$ and let $F$ be a subgroup of $\tilde{F}$. If for some $p, 0 \leqslant p \leqslant 1$, the (Weak) Corestriction Principle holds for the image of the coboundary map $\delta: \mathrm{H}^{p}(k, \bar{G}) \rightarrow \mathrm{H}^{p+1}(k, \tilde{F})$ then the same holds for the image of $\delta_{1}: \mathrm{H}^{p}(k, \bar{G}) \rightarrow \mathrm{H}^{p+1}(k, F)$. In particular, we have $e(p) \Leftrightarrow f(p)$.

Proof. We need only show that $f) \Rightarrow e)$. Let $G=\tilde{G} / F, F^{\prime}=\operatorname{Ker}(G \rightarrow \bar{G})$. Consider the following commutative diagram.

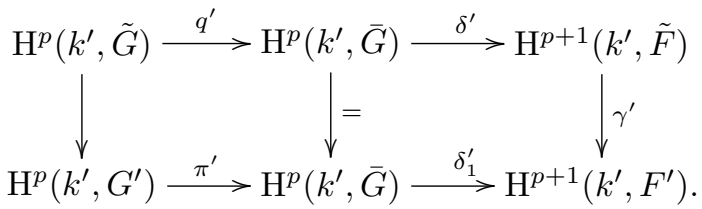

Recall that $\tilde{G}$ is the simply connected covering for both $\bar{G}$ and $G^{\prime}$.) One sees that $\delta_{1}^{\prime}=\gamma^{\prime} \delta^{\prime}$. Thus, if the Weak Corestriction Principle for images holds for $\delta$, then by Lemmas 1.1 and 1.5, the same holds for $\delta_{1}$.

Proposition 2.6. Assume that $a(p, q)$ holds for all $G$ with simply connected semisimple part $G^{\prime}$. Then $a(p, q)$ holds itself.

Proof. For $p=0$ the assertion follows easily by considering any $z$-extension of $G$. For $p=q=1$, it follows from Lemma 1.3 that for any finite extension $k^{\prime}$ of $k$ and any element $x \in \mathrm{H}^{1}\left(k^{\prime}, G\right)$, there exists a $x$-lifting $z$-extension of $\pi: G \rightarrow T$, all defined over $k$ :

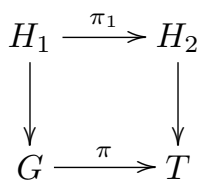

Here $H_{2}$ is a torus and $H_{1}$ has simply connected semisimple part. By assumption, the Weak Corestriction Principle holds for the image of the induced map $\pi_{1}^{*}: \mathrm{H}^{1}\left(k, H_{1}\right) \rightarrow \mathrm{H}^{1}\left(k, H_{2}\right)$. Let $\alpha: \mathrm{H}^{1}(k, G) \rightarrow \mathrm{H}^{1}(k, T)$ be the connecting map. By chasing on suitable diagrams one sees that the image of $x$ in $\mathrm{H}^{1}(k, T)$ via Cores $\circ \alpha$ lies in the subgroup generated by the image of $\mathrm{H}^{1}(k, G)$. Hence the Weak Corestriction Principle for images holds for $\alpha$.

The case $p=1, q=2$ is considered in a similar way. 
Proposition 2.7. Let $1 \rightarrow T \rightarrow G_{1} \stackrel{\pi}{\rightarrow} G \rightarrow 1$ be an exact sequence of $k$-groups, where $G$ is semisimple and $T$ is a central subgroup of a reductive $k$-group $G_{1}$. Let $G_{1}=G_{1}^{\prime} S_{1}$, where $G_{1}^{\prime}=\left[G_{1}, G_{1}\right], S_{1}$ is a central torus and $F:=S_{1} \cap G_{1}^{\prime}$. If for some $p, 0 \leqslant p \leqslant 1$, the (Weak) Corestriction Principle holds for the image of the coboundary map $\delta: \mathrm{H}^{p}(k, G) \rightarrow \mathrm{H}^{p+1}(k, F)$, then the same holds for the image of the coboundary map $\mathrm{H}^{p}(k, G) \rightarrow \mathrm{H}^{p+1}(k, T)$. In particular, $d(p) \Rightarrow c(p)$, i.e., c) $\Leftrightarrow d)$.

Proof. It follows from the assumption that $G_{1}$ is also connected and $\pi\left(G_{1}^{\prime}\right)=G$. It is clear that $T$ is a central subgroup of $G$ which contains $S_{1}$. We consider the following commutative diagram

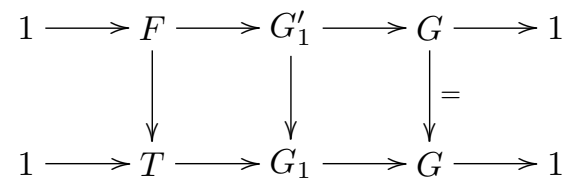

Now the proposition follows easily from this diagram as in the proof of Proposition 2.5, by combining with Lemmas 1.1 and 1.5 .

Proposition 2.8. Assume that for some $p, 0 \leqslant p \leqslant 1$, the (Weak) Corestriction Principle holds for the image of the connecting map $\mathrm{H}^{p}(k, H) \rightarrow \mathrm{H}^{p}(k, T)$ for any $k$-homomorphism $H \rightarrow T$ of connected reductive k-groups $H, T$, with $T$ a torus. Then the same holds for the image of the coboundary map $\mathrm{H}^{p}(k, G) \rightarrow \mathrm{H}^{p+1}\left(k, F^{\prime}\right)$ deduced from any isogeny $1 \rightarrow F^{\prime} \rightarrow G_{1} \rightarrow G \rightarrow 1$ with kernel $F^{\prime}$ and connected reductive $k$-group $G$, i.e., $a(p, p) \Rightarrow d(p)$. In particular, if a) holds then $d)$ holds.

Proof. To prove the assertion, we use the following Ono's crossed diagram (see [O] for details) which allows one to embed an exact sequence with finite kernel of multiplicative type (i.e., isogeny) into another one with induced $k$-torus as a kernel. We will denote all maps in the following diagrams (over $k$ and $k^{\prime}$ ) by the same symbols:

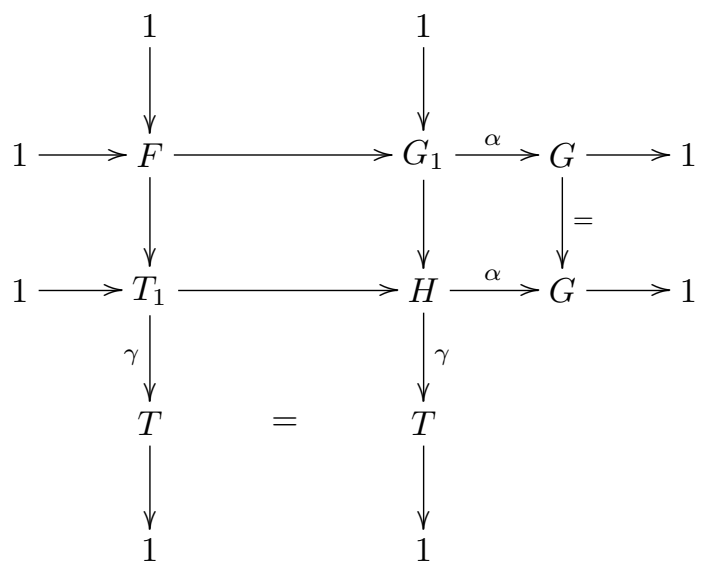


where $T_{1}$ is an induced $k$-torus. From this diagram we derive the following commutative diagram

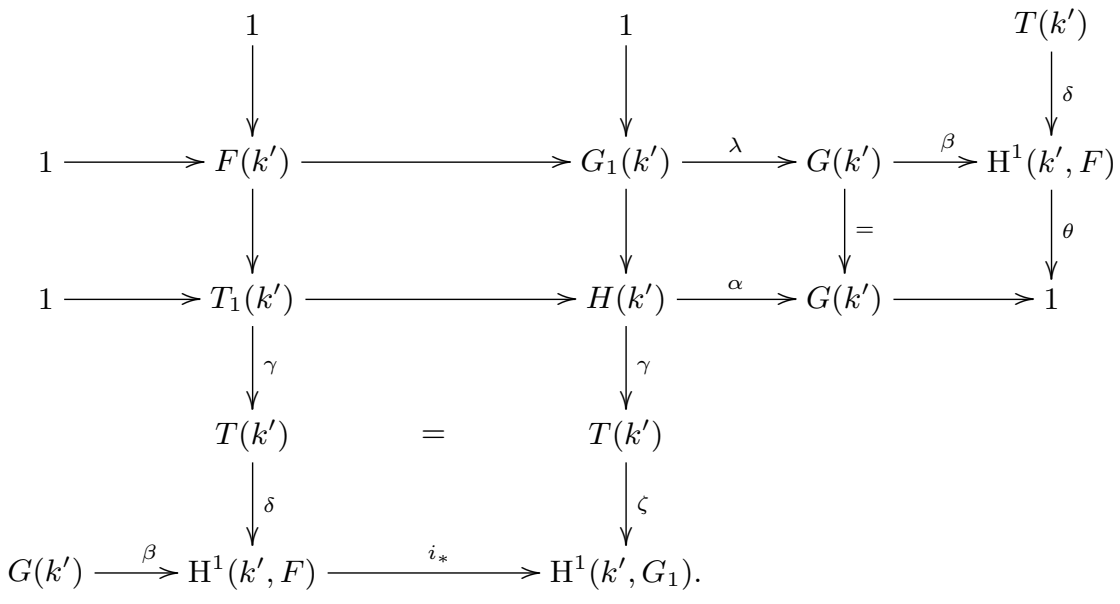

We need the following lemma due to Merkurjev, which is valid for any crossdiagram $(*)$ above, where $T$ is not necessarily an induced torus.

Lemma 2.8.1. [Me2] We have the following anti-commutative diagram

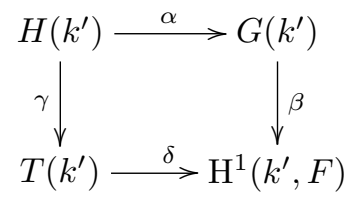

for any field extension $k \subset k^{\prime}$.

We continue the proof of Proposition 2.8 and we assume first that $p=0$. Note that in this case, the Weak Corestriction Principle is just the usual Corestriction Principle.

Since $T_{1}$ is an induced $k$-torus, $H^{1}\left(K, T_{1}\right)=0$ for any field extension $K / k$, so we have $\alpha\left(H\left(k^{\prime}\right)\right)=G\left(k^{\prime}\right)$. Now for any $g^{\prime} \in G\left(k^{\prime}\right)$, let $h^{\prime} \in H\left(k^{\prime}\right)$ such that $\alpha\left(h^{\prime}\right)=g^{\prime}$, and denote $t^{\prime}=\gamma\left(h^{\prime}\right), f^{\prime}=\beta\left(g^{\prime}\right)$. Since the diagram in Lemma 2.8.1 is anti-commutative, we have

$$
\begin{aligned}
\delta\left(t^{\prime}\right) & =\delta\left(\gamma\left(h^{\prime}\right)\right) \\
& =-\beta\left(\alpha\left(h^{\prime}\right)\right) \\
& =-f^{\prime} .
\end{aligned}
$$

Then for $f^{\prime}=\beta\left(g^{\prime}\right)$ we have $\theta\left(f^{\prime}\right)=0$ hence $y^{\prime}=\delta\left(z^{\prime}\right)$ for some $z^{\prime} \in T\left(k^{\prime}\right)$. The image $z \in T(k)$ of $z^{\prime}$ via $N_{k^{\prime} / k}: T\left(k^{\prime}\right) \rightarrow T(k)$ is such that $\delta(z)=f:=\operatorname{Cores}_{F}\left(f^{\prime}\right)$. 
Now look at the diagram on the left hand side. By assumption, the Corestriction Principle holds for the image of $H(k) \rightarrow T(k)$, so there are $h_{i} \in H(k)$ such that

$$
\sum_{i} \gamma\left(h_{i}\right)=t:=N_{k^{\prime} / k}\left(t^{\prime}\right) \text {. }
$$

Let $g=\alpha(h), g_{i}=\alpha\left(h_{i}\right)$. Then

$$
\begin{aligned}
\delta(t) & =\sum_{i} \delta\left(\gamma\left(h_{i}\right)\right) \\
& =-\sum_{i} \beta\left(\alpha\left(h_{i}\right)\right) \\
& =-\sum_{i} \beta\left(g_{i}\right) \\
& =\delta\left(N_{k^{\prime} / k}\left(t^{\prime}\right)\right) \\
& =\operatorname{Cores}_{F}\left(\delta\left(t^{\prime}\right)\right) .
\end{aligned}
$$

Since $\delta\left(t^{\prime}\right)=-f^{\prime}$ (see above) and $\delta\left(z^{\prime}\right)=f^{\prime}$, we have $\delta\left(t^{\prime} z^{\prime}\right)=0$ and $\operatorname{Cores}_{F}\left(\delta\left(t^{\prime} z^{\prime}\right)\right)=0$, so

$$
\begin{aligned}
\operatorname{Cores}_{F}\left(\delta\left(t^{\prime}\right)\right) & =-\operatorname{Cores}_{F}\left(\delta\left(z^{\prime}\right)\right) \\
& =-f .
\end{aligned}
$$

Therefore $f=\sum_{i} \beta\left(g_{i}\right)$, so $f \in\langle\operatorname{Im}(\beta)\rangle$ and the case $p=0$ is proved.

Now let $p=1$. For any finite extension $k^{\prime}$ of $k$ and for any element $g^{\prime}$ from $\mathrm{H}^{1}\left(k^{\prime}, G\right)$, by Lemma 1.2 we may choose a $g^{\prime}$-lifting $z$-extension

$$
1 \rightarrow T_{1} \rightarrow H \rightarrow G \rightarrow 1
$$

defined over $k$, such that there is an embeding $F \hookrightarrow T_{1}$. We consider the following diagram, which is similar to the one we have just considered, with the only difference that the dimension is shifted.

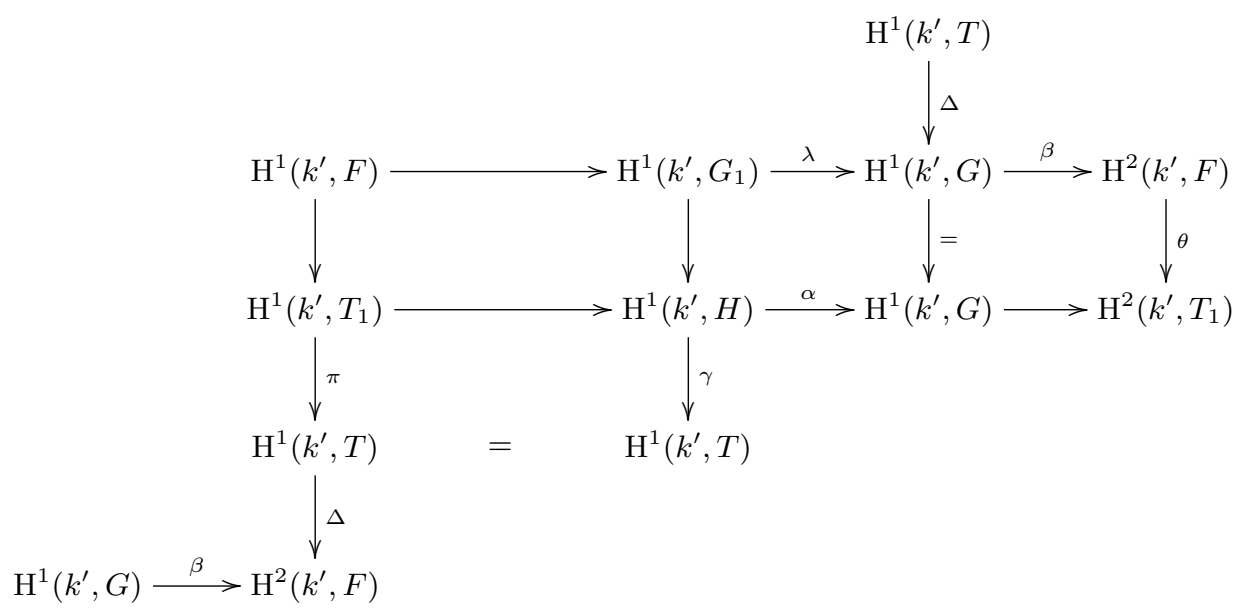

We need the following analog of 2.8.1 for higher dimension. 
Lemma 2.8.2. We have the following anti-commutative diagram for any cross$\operatorname{diagram}(*)$

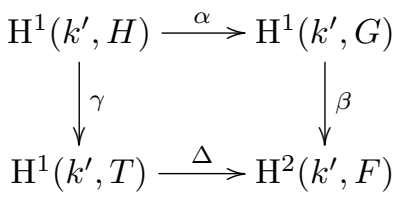

Proof. Let $h=\left[\left(h_{s}\right)\right] \in \mathrm{H}^{1}\left(k^{\prime}, H\right), g=\alpha(h) \in \mathrm{H}^{1}\left(k^{\prime}, G\right)$. Then $g=\left[\left(g_{s}\right)\right]$, where $g_{s}=\alpha\left(h_{s}\right)$. We choose for each $s$ an element $g_{s}^{\prime} \in G_{1}\left(k_{s}\right)$ such that $g_{s}=\alpha\left(g_{s}^{\prime}\right)$. Then

$$
h_{s}=g_{s}^{\prime} t_{s}, t_{s} \in T_{1}\left(k_{s}\right)
$$

One deduces from this

$$
f_{s r} g_{s r}^{\prime}=g_{s}^{\prime}{ }^{s} g_{r}^{\prime}\left(s, r \in \operatorname{Gal}\left(k_{s} / k^{\prime}\right)\right)
$$

for some $f_{s r} \in F\left(k_{s}\right)$ and we know (see [Se], Chap. I) that $\left(f_{s r}\right)$ is a 2-cocycle which is a representative of $\beta\left(\left[\left(g_{s}\right)\right]\right)$. From $h_{s}=g_{s}^{\prime} t_{s}$ we deduce that

$$
\gamma\left(h_{s}\right)=\gamma\left(g_{s}^{\prime}\right) \gamma\left(t_{s}\right)=\gamma\left(t_{s}\right),
$$

hence for $t=\left[\gamma\left(h_{s}\right)\right] \in \mathrm{H}^{1}\left(k^{\prime}, T\right)$ we have

$$
\Delta(t)=\left[\left(t_{s r}^{-1} t_{s}{ }^{s} t_{r}\right)\right] \in \mathrm{H}^{2}\left(k^{\prime}, F\right) .
$$

Now the product of two 2-cocycles is

$$
\left(t_{s r}^{-1} t_{s}{ }^{s} t_{r}\right)\left(g_{s r}^{\prime-1} g_{s}^{\prime}{ }^{s} g_{r}^{\prime}\right)=h_{s r}^{-1} h_{s}{ }^{s} h_{r}=1,
$$

since $\left(h_{s}\right)$ is a 1-cocycle. Thus

$$
\beta(\alpha(h))=-\Delta(\gamma(h))
$$

and the lemma follows.

With $g^{\prime} \in \mathrm{H}^{1}\left(k^{\prime}, G\right)$ as above, let $h^{\prime} \in \mathrm{H}^{1}\left(k^{\prime}, H\right)$ such that $g^{\prime}=\alpha\left(h^{\prime}\right)$. (Recall that $H$ is a $g^{\prime}$-lifting $z$-extension.) Take a cocycle representative $\left(g_{s}\right)$ of $g^{\prime}$ and let $g_{s}=\alpha\left(g_{1, s}\right), g_{1, s} \in G_{1}\left(k_{s}\right)$. Let $\left(h_{s}^{\prime}\right)_{s}$ be a representative of $h^{\prime}, h_{s}^{\prime} \in H\left(k_{s}\right)$. Then

$$
\begin{aligned}
\beta\left(g^{\prime}\right) & =\left[\left(g_{1, s t}^{-1} g_{1, s}{ }^{s} g_{1, t}\right)\right] \\
& =-\Delta\left(\gamma\left(h^{\prime}\right)\right)
\end{aligned}
$$

by the lemma above. Therefore

$$
\begin{aligned}
\operatorname{Cores}_{F}\left(\beta\left(g^{\prime}\right)\right) & =-\operatorname{Cores}_{F}\left(\Delta\left(\gamma\left(h^{\prime}\right)\right)\right) \\
& =-\Delta\left(\operatorname{Cores}_{T}\left(\gamma\left(h^{\prime}\right)\right)\right) .
\end{aligned}
$$

By assumption, we have $\operatorname{Cores}_{T}\left(\gamma\left(h^{\prime}\right)\right)=\Sigma_{i} \gamma\left(h_{i}\right)$ for some $h_{i} \in \mathrm{H}^{1}(k, H)$. Let $g_{i}=\alpha\left(h_{i}\right) \in \mathrm{H}^{1}(k, G)$. Then from above we have 


$$
\begin{aligned}
\operatorname{Cores}_{F}\left(\beta\left(g^{\prime}\right)\right) & =-\Delta\left(\operatorname{Cores}_{T}\left(\gamma\left(h^{\prime}\right)\right)\right) \\
& =-\Delta\left(\Sigma_{i} \gamma\left(h_{i}\right)\right) \\
& =-\Sigma_{i} \Delta\left(\gamma\left(h_{i}\right)\right) \\
& =\Sigma_{i} \beta\left(\alpha\left(h_{i}\right)\right)(\text { by }(* *)) \\
& =\Sigma_{i} \beta\left(g_{i}\right)
\end{aligned}
$$

as required.

For a connected reductive group $G$ we denote by $\operatorname{Ad}(G)$ the adjoint group of $G$, $\operatorname{Ad}(G)=G / \operatorname{Cent}(G)$.

Proposition 2.9. Let $\bar{G}$ be an adjoint semisimple k-group with fundamental group $\tilde{F}$.

a) $(p=0)$ Assume that Weak Corestriction Principle holds for the image of the coboundary map $\delta: \mathrm{H}^{0}(k, \bar{G}) \rightarrow \mathrm{H}^{1}(k, \tilde{F})$. Then the same holds for the connecting map $\alpha: \mathrm{H}^{0}(k, G) \rightarrow \mathrm{H}^{0}(k, T)$ for all connected reductive $k$-groups $G$, T, with $T$ a torus such that $\operatorname{Ad}(G)=\bar{G}$. In particular, if $f(0)$ holds then $a(0,0)$ holds.

b) $(p=1)$ Assume that Corestriction Principle holds for the image of the coboundary map $\Delta: \mathrm{H}^{1}(k, \bar{G}) \rightarrow \mathrm{H}^{2}(k, \tilde{F})$. Then the same holds for the connecting map $\mathrm{H}^{1}(k, G) \rightarrow \mathrm{H}^{1}(k, T)$ for all connected reductive groups $G$, $T$ with a torus $T$ such that $\operatorname{Ad}(G)=\bar{G}$.

Proof. a) Notice that in the case $p=0$, the Weak Corestriction Principle is just the Coretsriction Principle. Assume that we are given an exact sequence

$$
1 \rightarrow G_{1} \rightarrow G \rightarrow T \rightarrow 1
$$

of connected reductive $k$-groups with $T$ a torus. Let $G^{\prime}=[G, G], G=G^{\prime} . S$, where $S$ is a central torus of $G$. Denote $F^{\prime}=\operatorname{Cent}\left(G^{\prime}\right), F=G^{\prime} \cap S$, which are finite central subgroup of $G^{\prime}$. From Proposition 2.5 and its proof it follows that the Weak Corestriction Principle holds for the connecting map $\delta: \mathrm{H}^{0}(k, \bar{G}) \rightarrow \mathrm{H}^{1}\left(k, F^{\prime}\right)$.

Consider the following commutative diagram

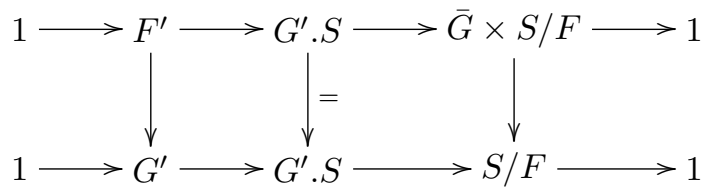

and also the following commutative diagram

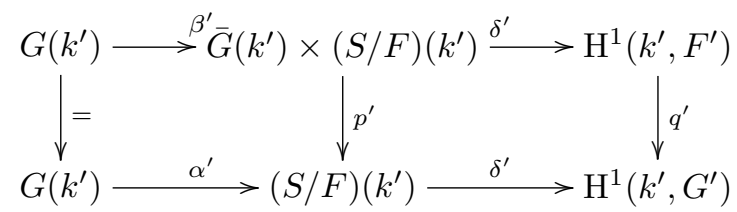


By our assumption, the Corestriction Principle holds for the image of $\delta^{\prime}$. We claim that the composition of the maps

$$
\bar{G}\left(k^{\prime}\right) \times(S / F)\left(k^{\prime}\right) \stackrel{p^{\prime}}{\rightarrow}(S / F)\left(k^{\prime}\right) \stackrel{\text { Cores }_{S / F}}{\rightarrow}(S / F)(k) \stackrel{\delta}{\rightarrow} \mathrm{H}^{1}\left(k, G^{\prime}\right),
$$

and that of the maps

$$
\bar{G}\left(k^{\prime}\right) \times(S / F)\left(k^{\prime}\right) \stackrel{\delta^{\prime}}{\rightarrow} \mathrm{H}^{1}\left(k^{\prime}, F^{\prime}\right) \stackrel{\text { Cores }_{F^{\prime}}}{\rightarrow} \mathrm{H}^{1}\left(k, F^{\prime}\right) \stackrel{q}{\rightarrow} \mathrm{H}^{1}\left(k, G^{\prime}\right)
$$

are the same. Indeed, denote by $p$ and $q$ the maps similar to $p^{\prime}$ and $q^{\prime}$, by considering the fields $k$ and $k^{\prime}$ interchanged. Then for $x=\left(g^{\prime}, s^{\prime}\right) \in \bar{G}\left(k^{\prime}\right) \times(S / F)\left(k^{\prime}\right)$ and $s=\operatorname{Cores}_{S / F}\left(s^{\prime}\right) \in(S / F)(k)$ we have

$$
\delta\left(\text { Cores }_{S / F}\left(p^{\prime}(x)\right)\right)=\delta\left(\text { Cores }_{S / F}\left(s^{\prime}\right)\right)=\delta(s) .
$$

By assumption, there is $g \in \bar{G}(k)$ such that

$$
\operatorname{Cores}_{F^{\prime}}\left(\delta^{\prime}\left(g^{\prime}\right)\right)=\delta(g)
$$

hence such that

$$
\operatorname{Cores}_{F^{\prime}}\left(\delta^{\prime}\left(g^{\prime}, s^{\prime}\right)\right)=\delta(g, s) .
$$

Since $p$ and $p^{\prime}$ are surjective and the above diagram is commutative, it follows that for $y=(g, s) \in \bar{G}(k) \times(S / F)(k)$ we have

$$
\begin{aligned}
\delta\left(\operatorname{Cores}_{S / F}\left(p^{\prime}(x)\right)\right) & =\delta(s) \\
& =\delta(p(g, s)) \\
& =q(\delta(g, s)) \\
& =q\left(\operatorname{Cores}_{F^{\prime}}\left(\delta^{\prime}\left(g^{\prime}, s^{\prime}\right)\right)\right) \\
& =q\left(\operatorname{Cores}_{F^{\prime}}\left(\delta^{\prime}(x)\right)\right)
\end{aligned}
$$

as claimed. Now the assertion of the theorem follows from the equality $\alpha^{\prime}=p^{\prime} \beta^{\prime}$. Indeed, let $x^{\prime} \in G\left(k^{\prime}\right), x^{\prime \prime}=\beta^{\prime}\left(x^{\prime}\right)=\left(g^{\prime}, s^{\prime}\right), y^{\prime}=\alpha^{\prime}\left(x^{\prime}\right), y=\operatorname{Cores}_{S / F}\left(y^{\prime}\right)$. Then $\alpha^{\prime}\left(x^{\prime}\right)=p^{\prime} \beta^{\prime}\left(x^{\prime}\right)=p^{\prime}\left(x^{\prime \prime}\right)$ hence

$$
\begin{aligned}
\delta\left(\operatorname{Cores}_{S / F}\left(p^{\prime}\left(x^{\prime \prime}\right)\right)\right) & =q\left(\operatorname{Cores}_{F^{\prime}}\left(\delta^{\prime}\left(x^{\prime \prime}\right)\right)\right) \\
& =q\left(\operatorname{Cores}_{F^{\prime}}\left(\delta^{\prime}\left(\beta^{\prime}\left(x^{\prime}\right)\right)\right)\right) \\
& =1,
\end{aligned}
$$

since $\delta^{\prime} \beta^{\prime}=0$. Therefore

$$
\text { Cores }_{S / F}\left(p^{\prime}\left(x^{\prime \prime}\right)\right) \in \operatorname{Ker}(\delta)=\operatorname{Im}(\alpha) .
$$

$b$ ) Now consider the case $p=1$. Let us be given an exact sequence

$$
1 \rightarrow G_{1} \rightarrow G \rightarrow T \rightarrow 1
$$


of connected reductive $k$-groups with $T$ a torus such that $\operatorname{Ad}(G)=\bar{G}$. From Proposition 2.5 and its proof it follows that the Corestriction Principle holds for $\mathrm{H}^{1}(k, \bar{G}) \rightarrow \mathrm{H}^{2}\left(k, F^{\prime}\right)$. We may use the above notation and consider the derived diagram of cohomology sets deduced from the diagram at the beginning of the proof, considered over $k$ and over $k^{\prime}$; we have the following exact sequences of cohomology :

$$
\begin{gathered}
\mathrm{H}^{1}\left(k, F^{\prime}\right) \rightarrow \mathrm{H}^{1}\left(k, G^{\prime}\right) \rightarrow \mathrm{H}^{1}(k, \bar{G}) \stackrel{\Delta_{1}}{\rightarrow} \mathrm{H}^{2}\left(k, F^{\prime}\right), \\
\mathrm{H}^{1}\left(k, F^{\prime}\right) \rightarrow \mathrm{H}^{1}\left(k, S F^{\prime}\right) \rightarrow \mathrm{H}^{1}(k, S / F) \stackrel{\Delta_{2}}{\longrightarrow} \mathrm{H}^{2}\left(k, F^{\prime}\right),
\end{gathered}
$$

and also similar sequences when $k$ is replaced by $k^{\prime}$ (where we put "'" on the corresponding maps). The following diagram is commutative with exact lines :

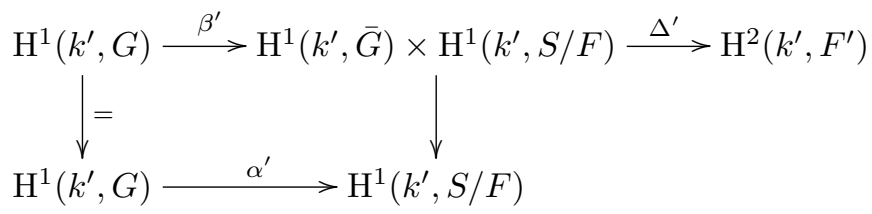

where the map $\Delta^{\prime}$ is such that

$$
\Delta^{\prime}(g, s)=\Delta_{1}^{\prime}(g)+\Delta_{2}^{\prime}(s),
$$

and the "+" is taken in $\mathrm{H}^{2}\left(k^{\prime}, F^{\prime}\right)$. Let $x^{\prime} \in \mathrm{H}^{1}\left(k^{\prime}, G\right), \beta^{\prime}\left(x^{\prime}\right)=\left(g^{\prime}, s^{\prime}\right), g^{\prime} \in$ $\mathrm{H}^{1}\left(k^{\prime}, \bar{G}\right), s^{\prime} \in \mathrm{H}^{1}\left(k^{\prime},(S / F)\right), s=$ Cores $_{k^{\prime} / k}\left(s^{\prime}\right) \in \mathrm{H}^{1}(k,(S / F))$. Then $\Delta^{\prime}\left(g^{\prime}, s^{\prime}\right)=$ 0 , so $\Delta_{2}^{\prime}\left(s^{\prime}\right)=-\Delta_{1}^{\prime}\left(g^{\prime}\right)$. By assumption, the Corestriction Principle holds for the image of $\Delta_{1}$, so via corestriction map we have

$$
\begin{aligned}
\operatorname{Cores}_{k^{\prime} / k}\left(\Delta_{2}^{\prime}\left(s^{\prime}\right)\right) & =\Delta_{2}(s) \\
& =\operatorname{Cores}_{k^{\prime} / k}\left(-\Delta_{1}^{\prime}\left(g^{\prime}\right)\right) \\
& =- \text { Cores }_{k^{\prime} / k}\left(\Delta_{1}^{\prime}\left(g^{\prime}\right)\right) \\
& =-\Delta_{1}(\bar{g})
\end{aligned}
$$

for some $\bar{g} \in \mathrm{H}^{1}(k, \bar{G})$. Therefore $\Delta(g, s)=0$, i.e., $(\bar{g}, s)=\beta(g), g \in \mathrm{H}^{1}(k, G)$, or equivalently $s=\alpha(g)$.

Remark. The same proof of Proposition 2.9, b) works also in the case $p=0$, so we have another proof of Proposition 2.9, a).

Finally, by summing up the results we proved above, we obtain the following theorem which is the main result of this section.

Theorem 2.10. 1) For Weak Corestriction Principle we have the following equivalent statements:

$$
a) \Leftrightarrow b), c) \Leftrightarrow d), e) \Leftrightarrow f) .
$$


2) We have the following interdependence between the statements a) - f) with particular values of $p$ and $q$.

a) For low dimension:

$$
a(0,1) \Leftarrow a(0,0) \Leftrightarrow b(0) \Leftrightarrow c(0) \Leftrightarrow d(0) \Leftrightarrow e(0) \Leftrightarrow f(0)
$$

b) For higher dimension:

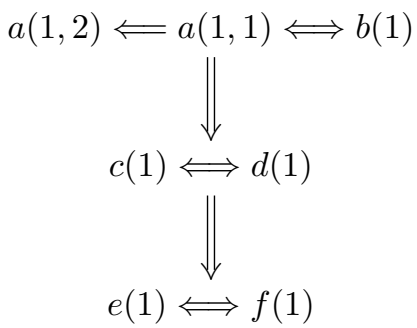

Proof. It follows from results proved above. We just indicate the logical dependence of these statements; the rest follows from this.

$b) \Rightarrow a)$ : see Proposition 2.1 .

$a) \Rightarrow b)$ : see Proposition 2.3 .

$a(0,0) \Rightarrow a(0,1), a(1,1) \Rightarrow a(1,2)$ : see Proposition 2.4.

$e) \Leftrightarrow f)$ : see Proposition 2.5.

c) $\Leftrightarrow d)$ : see Proposition 2.7 .

$a) \Rightarrow d)$ : see Proposition 2.8 .

$f(0) \Rightarrow a(0,0)$ : see Proposition 2.9, a). The first part of $b)$ also follows from this. The other equivalent relations follow from above ones.

If we consider the Corestriction Principle instead of Weak Corestriction Principle, then Theorem 2.10 still holds and we have stronger assertion as follows.

Theorem 2.11. For the Corestriction Principle, all the statements a) - f) are equivalent. More precisely we have the following:

a) For low dimension:

$$
a(0,1) \Leftarrow a(0,0) \Leftrightarrow b(0) \Leftrightarrow c(0) \Leftrightarrow d(0) \Leftrightarrow e(0) \Leftrightarrow f(0)
$$

b) For higher dimension:

$$
a(1,2) \Leftarrow a(1,1) \Leftrightarrow b(1) \Leftrightarrow c(1) \Leftrightarrow d(1) \Leftrightarrow e(1) \Leftrightarrow f(1)
$$

Proof. The proofs remain the same, by combining with Theorem 2.10, b).

Corollary 2.12. The Corestriction Principle in higher dimension (i.e. the statements $a(1,1), b(1), c(1), d(1), e(1), f(1))$ does not hold in general.

Proof. The example given by Rosset and Tate shows that the Corestriction Principle for connecting map $\mathrm{H}^{1}\left(k, \mathrm{PGL}_{n}\right) \rightarrow \mathrm{H}^{2}\left(k, \mu_{n}\right)$ does not hold true in general. So by Theorem 2.11, b), in general, this is neither true for connecting maps $\mathrm{H}^{1}(k, G) \rightarrow$ $\mathrm{H}^{1}(k, T)$, where $G, T$ are reductive groups and $T$ is a diagonalisable group, i.e., $a(1,1)$ does not hold. The other cases follow in the same way. 
Remark 2.13. 1) It is still an open question if the Corestriction Principle is true for lower dimension (in which case it coincides with the Weak Corestriction Principle). There are some cases, where the Corestriction Principle holds. For example, if $G$ is a connected reductive $k$-group and has trivial group $G(k) / R$ of $R$-equivalence classes over $k$, then the Corestriction Principle holds for any connecting homomorphism $\mathrm{H}^{0}(k, G) \rightarrow \mathrm{H}^{0}(k, T)$, with $T$ a torus (see [Me2]); or if $G$ is semisimple and $G(k) / R=1$ then the Corestriction Principle holds for the coboundary map $\mathrm{H}^{0}(k, G) \rightarrow \mathrm{H}^{1}(k, F)$, where $F$ is the kernel of an isogeny of semisimple groups $1 \rightarrow F \rightarrow G_{1} \rightarrow G \rightarrow 1$ (see [Gi]). In [T2] we show that the Corestriction Principle holds for the images and kernels of connecting maps over local or global fields of characteristic 0 . This result can be considered as a cohomological counterpart of results of Lenstra and Tate.

2) If the group $T$ in the statement $c$ ) is connected (i.e. a torus), then one may use the functoriality of the map $a b_{G}: \mathrm{H}^{1}(k, G) \rightarrow \mathrm{H}_{a b}^{1}(k, G)$ to deduce $b(p) \Rightarrow c(p)$. However, this does not seem to be applicable to the general case, so the proof of $a) \Rightarrow d)$ seems to be inevitable. Regarding Weak Corestriction Principle, it is not clear whether the implication $f(1) \Rightarrow a(1,1)$ always holds. In the next section we discuss the validity of the (weakest) condition $f$ ) above.

\section{A reduction to quasi-split case}

Let $G$ and $T$ be reductive groups defined over a field $k$ of characteristic 0 , where $G$ is connected and $T$ is commutative. In this section we are interested in Weak Corestriction Principle for the image of the coboundary map

$$
\Delta: \mathrm{H}^{1}(k, G) \rightarrow \mathrm{H}^{2}(k, T) .
$$

This map is induced from the exact sequence of $k$-groups

$$
1 \rightarrow T \rightarrow G_{1} \stackrel{\pi}{\rightarrow} G \rightarrow 1,
$$

and we assume $G_{1}$ to be connected (and reductive) and $T$ to be a central subgroup of $G_{1}$. Our goal is to reduce the proof of the Weak Corestriction Principle to the case of quasi-split groups.

By the way, we would like to mention the following reductive version of the Steinberg's Theorem. It will not be used anywhere in the sequel, but it is related to what we are going to do. Denote by $\Gamma$ the Galois group $\operatorname{Gal}(\bar{k} / k)$, and $\Gamma^{\prime}$ the Galois group $\operatorname{Gal}\left(\bar{k} / k^{\prime}\right)$, where $k^{\prime}$ is a finite extension of $k$.

Proposition 3.1. Let $G$ be a quasi-split connected reductive group defined over a field $k$ with connected center $S$, and let $\left(x_{s}\right)_{s \in \Gamma}$ be a 1-cocycle $\in Z^{1}(\Gamma, G)$. There exists a maximal $k$-torus $T_{0}$ of $G$ such that the cocycle $x_{s}$ is the product of two 1-cocycles $\left(y_{s}\right)$ and $\left(z_{s}\right)$ of $\Gamma$, where $\left(y_{s}\right)$ is a 1-cocycle cohomologous to one with values in $S$ and $\left(z_{s}\right)$ is a cocycle with values in $T_{0}$. Thus if the connected center $S$ of $G$ has trivial 1-cohomologies, then every 1-cocycle with values in $G$ is cohomologous to one with values in a maximal $k$-torus of $G$.

Proof. We consider the following exact sequence

$$
1 \rightarrow S \rightarrow G \stackrel{\pi}{\rightarrow} G^{\prime} \rightarrow 1
$$


where $G^{\prime}$ is semisimple and its derived sequence of cohomology

$$
\mathrm{H}^{1}(k, S) \rightarrow \mathrm{H}^{1}(k, G) \stackrel{\pi^{\prime}}{\rightarrow} \mathrm{H}^{1}\left(k, G^{\prime}\right) .
$$

Let $x$ be the class of $\left(x_{s}\right)$ in $\mathrm{H}^{1}(k, G)$. Since $G^{\prime}$ is semisimple and quasi-split over $k$, from the Steinberg's Theorem [St2], it follows that there is a maximal $k$-torus $T^{\prime}$ of $G^{\prime}$ and $\left(t_{s}^{\prime}\right) \in Z^{1}\left(\Gamma^{\prime}, T^{\prime}\right)$ such that $\pi^{\prime}\left(x_{s}\right)=g^{\prime-1} t_{s}^{\prime}{ }^{s} g^{\prime}$ for all $s \in \Gamma^{\prime}$ and $g^{\prime} \in G^{\prime}$. Let $t^{\prime}$ be the class of $\left(t_{s}^{\prime}\right)$ and $i: \mathrm{H}^{1}\left(k, T^{\prime}\right) \rightarrow \mathrm{H}^{1}\left(k, G^{\prime}\right)$. Then for the coboundary map $\Delta: \mathrm{H}^{1}\left(k, G^{\prime}\right) \rightarrow \mathrm{H}^{2}(k, S)$ we have

$$
\Delta\left(t^{\prime}\right)=\Delta\left(i\left(t^{\prime}\right)\right)=\Delta\left(\pi^{\prime}(x)\right)=0 .
$$

Hence $t^{\prime} \in \operatorname{Im}\left(\mathrm{H}^{1}\left(k, T_{0}\right) \stackrel{\pi^{\prime}}{\rightarrow} \mathrm{H}^{1}\left(k, T^{\prime}\right)\right)$, where $T_{0}$ is the preimage of $T^{\prime}$ in $G$, which is a maximal $k$-torus of $G$ and we use the same notation $\pi^{\prime}$ to denote the induced map of cohomologies. Let $t^{\prime}=\pi^{\prime}(t), t \in \mathrm{H}^{1}\left(k, T_{0}\right)$ so $\pi^{\prime}(i(t))=\pi^{\prime}(x)$. Let $G_{0}$ be the group $G$ twisted with the cocycle representing $i(t)$. This twisting does not effect the tori $S$ and $T_{0}$. Denote by $\tau_{t}$ the bijection $\mathrm{H}^{1}(k, G) \rightarrow \mathrm{H}^{1}\left(k, G_{0}\right)$. From the exact sequence $1 \rightarrow S \rightarrow G_{0} \rightarrow G_{0} / S \rightarrow 1$ we derive that the image $x_{1}$ of $x$ via $\tau_{t}$ has trivial image in $\mathrm{H}^{1}\left(k, G_{0} / S\right)$, so it lies in the image $\operatorname{Im}\left(\mathrm{H}^{1}(k, S) \rightarrow \mathrm{H}^{1}\left(k, G_{0}\right)\right)$. Let $g \in G_{0}$ (=G as a set) such that $x_{1, s}=g^{-1} p_{s}{ }^{s} g$ for all $s \in \Gamma^{\prime}$, where $\left(p_{s}\right)$ is a cocycle with values in $S$. Since $x_{1, s}=x_{s} t_{s}^{-1}$ (by definition of $\tau_{t}$, see [Se], Chap. I) we have

$$
x_{s}=g^{-1} p_{s}{ }^{s} g t_{s}
$$

as required.

The last statement follows from the fact that the map $\pi^{\prime}: \mathrm{H}^{1}(k, G) \rightarrow \mathrm{H}^{1}(k, G / S)$ is injective (by using the twisting argument).

We now show how to reduce the statement $a(1,2)$ of Section 2.0 , that the Weak Corestriction Principle holds for the image of the coboundary map $\mathrm{H}^{1}(k, G) \rightarrow$ $\mathrm{H}^{2}(k, T)$, where $G$ is a connected reductive group and $T$ is a central diagonalizable group, to the same statement in the quasi-split case. Consider the coboundary map $\mathrm{H}^{1}(k, G) \rightarrow \mathrm{H}^{2}(k, T)$ where $G=G_{1} / T$ is the quotient of a connected, reductive $k$-group $G_{1}$, and $T$ is a central $k$-subgroup of $G_{1}$. Denote by $G^{q}$ the unique quasisplit $k$-form of $G$, such that $G$ can be obtained from $G^{q}$ by inner twisting with an 1-cocycle $g \in Z^{1}\left(\Gamma, G^{q}\right)$. This twisting does not affect the group $T$, so we have the coboundary maps $\Delta: \mathrm{H}^{1}(k, G) \rightarrow \mathrm{H}^{2}(k, T), \Delta_{q}: \mathrm{H}^{1}\left(k, G^{q}\right) \rightarrow \mathrm{H}^{2}(k, T)$.

Proposition 3.2. With above notation, the image of $\Delta$ and that of $\Delta_{q}$ genarate the same subgroup of $\mathrm{H}^{2}(k, T)$. Therefore, if the Weak Corestriction Principle holds for the image of $\Delta_{q}$ then it also holds for that of $\Delta$.

Corollary 3.2.1. If the Weak Corestriction Principle holds for the image of coboundary maps $\Delta_{q}: \mathrm{H}^{1}\left(k, G^{q}\right) \rightarrow \mathrm{H}^{2}(k, T)$ for all quasi-split groups $G^{q}$ and diagonalizable group $T$, then it also holds in general.

Proof of Proposition 3.2.. We have the following commutative diagram, where all vertical maps are bijections (the "translation maps") (see [Se], Chap. I, Prop. 44) 


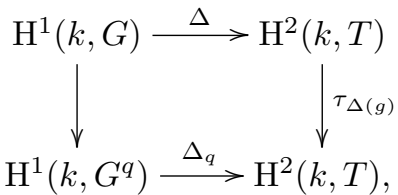

and similar diagram over any field extension of $k$. Since the image of $\Delta$ and $\Delta_{q}$ contains 0 (the neutral element of the group $\mathrm{H}^{2}(k, T)$ ), and the right vertical map is just the translation by the element $\Delta(g)$, we see that

$$
\Delta(g) \in D_{q}(k):=\left\langle\operatorname{Im}\left(\Delta_{q}\right)\right\rangle .
$$

By symmetry, we have

$$
\Delta(g) \in D(k):=\langle\operatorname{Im}(\Delta)\rangle .
$$

Therefore $D(k)=D_{q}(k)$ and it is also true for any field extension of $k$ and the assertion follows.

\section{Weak Corestriction Principle : adjoint semi-simple groups}

In this section we prove our second main result mentioned in Introduction. From Theorem 2.10 it follows that in order to prove the Weak Corestriction Principle for the image of a coboundary map $\mathrm{H}^{1}(k, G) \rightarrow \mathrm{H}^{2}(k, T)$, where $G$ is semisimple and $T$ is a diagonalizable group, one first tries to prove it for the coboundary map

$$
\mathrm{H}^{1}(k, G) \stackrel{\Delta}{\rightarrow} \mathrm{H}^{2}(k, F),
$$

where $G=G_{1} / F$ is the adjoint $k$-group of a semisimple $k$-group $G_{1}$ and $F$ is the center of $G_{1}$. Our main result of this section is the following. We refer the reader to [Ti] for various notions and properties of Tits indices.

Theorem 4.1 (Weak Corestriction Principle). With above notation, assume that $G$ is an adjoint semisimple $k$-group and contains only almost simple factors of classical and inner types ${ }^{1} \mathrm{~A}, \mathrm{~B}, \mathrm{C},{ }^{1} \mathrm{D}_{n}$ ( $n$ even). Assume further that $k$ contains $(m+1)$ th-roots of unity if $G$ contains a factor of type ${ }^{1} \mathrm{~A}_{m}$. Then for any finite extension $k^{\prime} / k$ the Weak Corestriction Principle holds for the image of $\Delta: \mathrm{H}^{1}(k, G) \rightarrow \mathrm{H}^{2}(k, F)$, i.e., for $\Delta^{\prime}: \mathrm{H}^{1}\left(k^{\prime}, G\right) \rightarrow \mathrm{H}^{2}\left(k^{\prime}, F\right)$ we have

$$
\operatorname{Cores}_{k^{\prime} / k}\left(\operatorname{Im}\left(\Delta^{\prime}\right)\right) \subset\langle\operatorname{Im}(\Delta)\rangle \text {. }
$$

In fact, most of interesting coboundary maps $\mathrm{H}^{1}(k, G) \rightarrow \mathrm{H}^{2}(k, F)$, where $G$ is semisimple and $F^{\prime}$ is finite, arise as coboundary maps deduced from the exact sequence

$$
1 \rightarrow F \rightarrow \tilde{G} \rightarrow \bar{G} \rightarrow 1,
$$

where $\tilde{G}$ is simply connected and semisimple, $\bar{G}$ is adjoint and $F=\tilde{F}$ is the center of $\tilde{G}$. Denote by $\Delta$ the coboundary map

$$
\mathrm{H}^{1}(k, G) \stackrel{\Delta}{\rightarrow} \mathrm{H}^{2}(k, \tilde{F}) .
$$


It follows from Theorem 2.10 (the equivalence $(e) \Leftrightarrow(f)$ ) that Theorem 4.1 is equivalent to the following.

Theorem (4.1'). With above notation, assume that $G$ is an adjoint semisimple $k$-group and contains only almost simple factors of classical and inner types ${ }^{1} \mathrm{~A}, \mathrm{~B}$, $\mathrm{C},{ }^{1} \mathrm{D}_{n}$ (n even) and that $k$ contains $(m+1)$ th-roots of unity if $G$ contains a factor of type ${ }^{1} \mathrm{~A}_{m}$. Then for any finite extension $k^{\prime} / k$, the Weak Corestriction Principle holds for the image of $\Delta: \mathrm{H}^{1}(k, G) \rightarrow \mathrm{H}^{2}(k, \tilde{F})$, i.e., for $\Delta^{\prime}: \mathrm{H}^{1}\left(k^{\prime}, G\right) \rightarrow \mathrm{H}^{2}\left(k^{\prime}, \tilde{F}\right)$ we have

$$
\operatorname{Cores}_{k^{\prime} / k}\left(\operatorname{Im}\left(\Delta^{\prime}\right)\right) \subset\langle\operatorname{Im}(\Delta)\rangle .
$$

Proof. The proof will be divided into few parts. It is clear that we may assume $G$ to be (absolutely) almost simple. By Proposition 3.2 we may assume also that $G$ is quasi-split, hence also split $k$-group (recall that $G$ is of inner type). From now on we assume that $G$ is an adjoint almost simple split $k$-group with simply connected covering $\tilde{G}$ and fundamental group $\tilde{F}$ (= center of $\tilde{G}$ ).

We will consider separately the type of the group $G$. Type ${ }^{1} \mathrm{~A}_{n}$.

It is proved by Rosset and Tate [RS], or follows directly from Merkurjev - Suslin Theorem ([MS]) (see Introduction).

Type $\mathrm{B}_{n}$.

We have the following

Proposition 4.2. Let $G$ be an adjoint absolutely almost simple $k$-group of type $\mathrm{B}_{n}$. Then the image of $\Delta$ generates $\mathrm{H}^{2}(k, \tilde{F})$ as a group. In particular, the Weak Corestriction Principle holds for the image of $\Delta$.

Proof. The Tits index of $G$ is as follows

$$
1 \circ--^{2} \circ \cdots \circ--^{n-1} \circ \Rightarrow \circ^{n}
$$

where the $i$-th vertex corresponds to the $\operatorname{root} \alpha_{i}$ of $\tilde{G}$ with respect to a fixed maximal $k$-split torus of $\tilde{G}$. The center $\tilde{F}$ of $\tilde{G}$ is given by

$$
\tilde{F}=\left\langle h_{n}(-1)\right\rangle \simeq \mu_{2},
$$

where $h_{i}(t), i=1, \ldots, n$ are multiplicative one-parameter subgroups of $\tilde{G}$ constructed in [St1] (see Appendix). Then the regular $k$-subgroup $\tilde{H}$ of $\tilde{G}$ with root system $\left\{\alpha_{n}\right\}$ is of type $\mathrm{A}_{1}$ and $k$-isomorphic to $\mathrm{SL}_{2}$ and contains $\tilde{F}$ as its center. Let $H=\tilde{H} / \tilde{F}$. Then from the commutative diagram

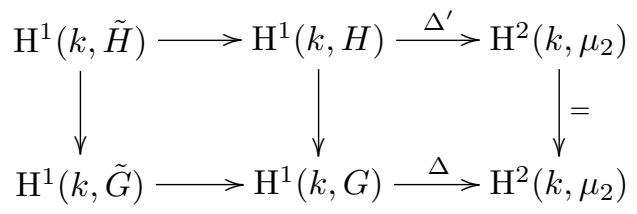

and the fact that the image of $\Delta^{\prime}$ generates the whole group $\mathrm{H}^{2}\left(k, \mu_{2}\right)={ }_{2} B r(k)$ by Merkurjev's Theorem [Me1], it follows that the same is true for $\Delta$. 


\section{Type $\mathrm{C}_{n}$.}

Proposition 4.3. Let $G$ be of type $\mathrm{C}_{n}$. Then the image of $\Delta$ generates $\mathrm{H}^{2}(k, \tilde{F})$ as a group. In particular, the Weak Corestriction Principle holds for the image of $\Delta$.

Proof. Since we may assume that $G$ is $k$-split, we have $G \simeq \operatorname{PSp}_{2 n}:=\operatorname{Sp}_{2 n} /\langle \pm 1\rangle$. It is well-known that $\mathrm{H}^{1}(k, G)$ classifies central simple $k$-algebras $A$ of degree $2 n$ with involution $\sigma$ of symplectic type (see [KMRT], p. 404). In particular, via the map $\Delta$, such a pair $(A, \sigma)$ is mapped to the class of $A$ in the Brauer group of $k$. Since any quaternion division algebra over $k$ is equipped with standard involution which is of symplectic type, while the quaternion algebras generate the whole group ${ }_{2} B r(k)$, the assertion is proved.

Type ${ }^{1} \mathrm{D}_{n}$.

Let $G(k)=\mathrm{PGU}^{+}(\Phi, D)$, where $\Phi$ is a non-degenerate skew-hermitian form with values in a division algebra $D$ with center $k$. Here we use the same notation as in [KMRT], Sec. 29. Denote by $G^{\prime}=\mathrm{SU}(\Phi)$ the corresponding special unitary $k$-group of $\Phi$ and $\tilde{G}=\operatorname{Spin}(\Phi)$. Let $F_{1}=\operatorname{Ker}\left(\tilde{G} \rightarrow G^{\prime}\right), F_{2}=\tilde{F} / F_{1}$. So $F_{i} \simeq \mu_{2}, i=1,2$, and there are coboundary maps

$$
\begin{aligned}
& \Delta_{1}: \mathrm{H}^{1}\left(k, G^{\prime}\right) \rightarrow \mathrm{H}^{2}\left(k, F_{1}\right), \\
& \Delta_{2}: \mathrm{H}^{1}(k, G) \rightarrow \mathrm{H}^{2}\left(k, F_{2}\right) .
\end{aligned}
$$

First we investigate the images of $\Delta_{i}$.

Proposition 4.4. With notation as above, the image of $\Delta_{1}$ generates $\mathrm{H}^{2}\left(k, F_{1}\right)$ as a group.

Proof. It follows from Proposition 3.2 that we may assume $G$ to be $k$-split, so we assume that $G^{\prime}=\mathrm{SO}(q)$, where $q=n\langle 1,-1\rangle$. Then it is well-known that $\mathrm{H}^{1}\left(k, G^{\prime}\right)$ classifies up to equivalence non-degenerate $2 n$-dimensional quadratic forms $q^{\prime}$ of the same determinant $(-1)^{n}$ as $q$ (see, e.g. [KMRT], Sec. 29). Also the map $\Delta_{1}$ maps each equivalence class $\left[q^{\prime}\right]$ to $w_{2}\left(\left[q^{\prime}\right]\right)-w_{2}([q])$, where $w_{2}$ denotes the Hasse - Witt invariant of the form. We denote by $(a, b)$ the standard quaternion algebra over $k$ with the basis $1, i, j, i j$, such that $i^{2}=a, j^{2}=b, i j=-j i$, and by $[(a, b)]$ its class in the Brauer group of $k$. With the following labelling in the Tits index of $G$

$$
\circ^{1}--\circ^{2}--\circ--\cdots--\circ^{q-1}--\circ^{q}--\circ--\cdots{ }^{n-2} \circ\left\langle_{\circ^{n-1}}^{\circ^{n}}\right.
$$

let $\tilde{H}$ be the regular $k$-subgroup of $\tilde{G}$ with root system $\left\{\alpha_{n-1}, \alpha_{n}\right\}$. Then we know (see Appendix) that the center of $\tilde{G}$ is given by

$$
\tilde{F}=\left\langle z_{1}, z_{2}\right\rangle
$$

with

$$
\begin{gathered}
z_{1}=h_{1}(-1) h_{3}(-1) \cdots h_{n-3}(-1) h_{n-1}(-1), \\
z_{2}=h_{1}(-1) h_{3}(-1) \cdots h_{n-3}(-1) h_{n}(-1)
\end{gathered}
$$


if $n$ is even and by

$$
\tilde{F}=\langle z\rangle
$$

with

$$
z=h_{1}(-1) h_{3}(-1) \cdots h_{n-2}(-1) h_{n-1}(i) h_{n}(-i),
$$

$\left(i^{2}=-1\right)$, if $n$ is odd. In both cases, we have

$$
F_{1}=\left\langle h_{n-1}(-1) h_{n}(-1)\right\rangle \subset \tilde{H} .
$$

We have the following commutative diagram where all rows are exact sequences

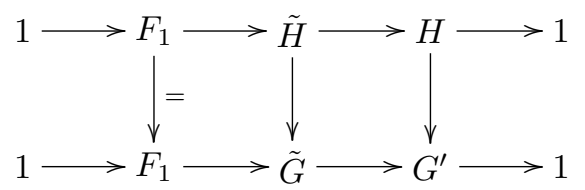

Therefore it suffices to show that the image of the connecting map

$$
\Delta_{1}^{\prime}: \mathrm{H}^{1}(k, H) \rightarrow \mathrm{H}^{2}\left(k, F_{1}\right)
$$

generates $\mathrm{H}^{2}\left(k, F_{1}\right)$ as a group. Since $\tilde{H}$ is $k$-split and of type $\mathrm{A}_{1} \times \mathrm{A}_{1}$, we have

$$
\tilde{H} \simeq \operatorname{Spin}(f), H \simeq \mathrm{SO}(f),
$$

where $f$ is a non-degenerate 4-dimensional quadratic form over $k$ of maximal Witt index. Then we have

$$
\Delta_{1}^{\prime}\left(\left[f^{\prime}\right]\right)=w_{2}\left(\left[f^{\prime}\right]\right)-w_{2}([f]),\left[f^{\prime}\right] \in \mathrm{H}^{1}(k, \mathrm{SO}(f)) .
$$

Let

$$
f^{\prime}=\langle a, b, c, d\rangle, a, b, c, d \in k, a b c d \neq 0, f=\langle 1,-1,1,-1\rangle .
$$

Since $\operatorname{det}\left(f^{\prime}\right)=\operatorname{det}(f)$, we may assume that $d=a b c$. We have thus

$$
\begin{aligned}
\Delta_{1}^{\prime}\left(\left[f^{\prime}\right]\right) & =[(a, b) \otimes(a, c) \otimes(a, a b c) \otimes \otimes(b, c) \otimes(b, a b c) \otimes(c, a b c)]-w_{2}([f]) \\
& =\left[\left(a, a b^{2} c^{2}\right) \otimes\left(b, a b c^{2}\right) \otimes(c, a b c)\right]-w_{2}([f]) \\
& =[(a, a) \otimes(b, a b) \otimes(c, a b c)]-w_{2}([f]) \\
& =[(a, a)]+[(b, a b)]+[(c, a b c)]-[(-1,-1)] .
\end{aligned}
$$

By taking $a=-1$ we get

$$
\Delta_{1}^{\prime}\left(\left[f^{\prime}\right]\right)=[(c,-b c)] .
$$

When $b, c$ run over $k^{*}$, it is clear that $(c,-b c)$ runs over all quaternion algebras. Hence by Merkurjev's Theorem [Me1], the image of $\Delta_{1}^{\prime}$ generates the 2-torsion subgroup $\mathrm{H}^{2}\left(k, F_{1}\right)={ }_{2} \operatorname{Br}(k)$.

Proposition 4.5. With above notation, if $n$ is even then the image of $\Delta_{2}$ generates $\mathrm{H}^{2}\left(k, F_{2}\right)$. 
Proof. Again by Proposition 3.2, since $G$ is of inner type, we may assume that $G$ is $k$ split, $G=\mathrm{PGO}^{+}(f)$, where $f$ is a non-degenerated quadratic form of dimension $2 n$, $\mathrm{PGO}^{+}(f)$ is the identity component of $\mathrm{PGO}(f)$. We have the following commutative diagram

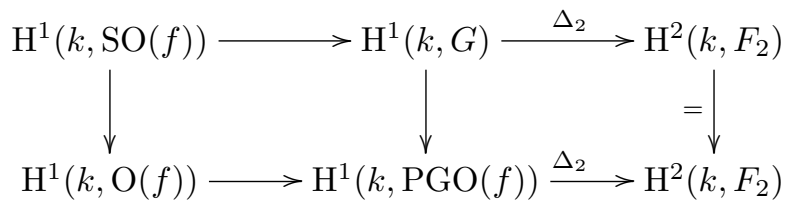

It is well-known that (see e.g. [KMRT], Sec. 29) $\mathrm{H}^{1}(k, \mathrm{PGO}(f)$ (resp. $\left.\mathrm{H}^{1}\left(k, \mathrm{PGO}^{+}(f)\right)\right)$ classifies central simple algebras $A$ of degree $2 n$ with center $k$ and involution $\sigma_{A}$ of orthogonal type (resp. such that $\left.\operatorname{det}\left(\sigma_{A}\right)=d:=\operatorname{det}(f)\right)$. The map $\Delta_{2}$ maps the isomorphism class $\left[\left(A, \sigma_{A}\right)\right]$ to the class $[A]$ of $A$ in the Brauer group ${ }_{2} B r(k)$.

Now assume that $n$ is even, $n=2 m$ so $d=1$. Take any quaternion division algebra $D$ over $k$ with standard involution $J$ and any $J$-skew element $\alpha \in D^{*}$. Consider the central simple algebra $A=M_{m}(D)$ with $J$-skew-hermitian form $\Phi=$ $\langle\alpha, \ldots, \alpha\rangle$ of dimension $m$ and the involution $\sigma_{A}$ on $A$ corresponding to $\Phi$. Then the image of $\left(A, \sigma_{A}\right)$ via $\Delta_{2}$ is equal to $[D]$. Therefore the assertion is proved.

Proposition 4.6. Let $G$ be of type ${ }^{1} \mathrm{D}_{n}, n$ is even. Then the Weak Corestriction Principle holds for the image of $\Delta$.

Proof. We show that the image of $\Delta$ generates $\mathrm{H}^{2}(k, \tilde{F})$ as a group. Consider the following commutative diagram

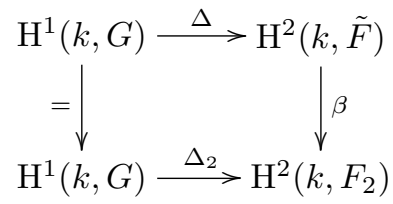

and similar one over the field $k^{\prime}$, where the corresponding maps will be denoted by the same symbols but with '. Let $x \in \mathrm{H}^{2}(k, \tilde{F})$. Then its image $\beta(x)$ is belong to the subgroup generated by the image of $\Delta_{2}$ by Prop. 4.5 . From the above diagram it follows that

$$
x \equiv\left(\Delta\left(g_{1}\right)+\cdots+\Delta\left(g_{m}\right)\right)(\bmod . \operatorname{Ker}(\beta)) .
$$

We have the following commutative diagram

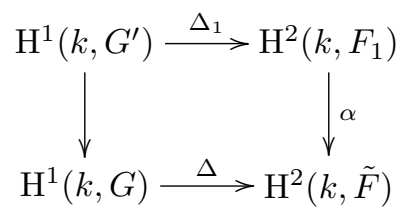


By Proposition 4.5 , the image of $\Delta_{1}$ generates $\mathrm{H}^{2}\left(k, F_{1}\right)$, so from the last diagram we conclude that the image of $\Delta$ generates $\mathrm{H}^{2}(k, \tilde{F})$. This is also true for any field, containing $k$. Therefore in this case the Weak Corestriction Principle holds and the proposition is proved.

Summing up, we have proved Theorem 4.1' hence also Theorem 4.1.

Remark 4.7. 1) If the field $k$ does not contain the $n$-roots of unity, as far as we know, the case ${ }^{1} \mathrm{~A}_{n}$ above is still open. Moreover, if denote by $D(n)(k)$ the subgroup of ${ }_{n} \mathrm{Br}(k)$ generated by the image of the connecting map

$$
\Delta: \mathrm{H}^{1}\left(k, \mathrm{PGL}_{n}\right) \rightarrow \mathrm{H}^{2}\left(k, \mu_{n}\right) \simeq{ }_{n} B r(k),
$$

and by $C(n)(k)$ the subgroup of ${ }_{n} B r(k)$ generated by cyclic algebras over $k$ of degree $n$, then we have the following inclusions

$$
C(n)(k) \subset D(n)(k) \subset{ }_{n} B r(k) .
$$

In [Me3] - [Me4], Merkurjev discussed the (still open in general up to now) conjecture that for any field $k$ we have $C(n)(k)={ }_{n} B r(k)$, i.e., the Brauer group of a field is generated by cyclic algebras, and proved some related results about validity of this conjecture. Our conjectures regarding the Weak Corestriction Principle in this case are that

a) For any finite extension $k^{\prime} / k$, we have $\operatorname{Cores}_{k^{\prime} / k}\left(D(n)\left(k^{\prime}\right)\right) \subset D(n)(k)$.

b) $D(n)(k)={ }_{n} B r(k)$.

The first conjecture follows from the second, which in turn follows from the one considered by Merkurjev above. Also b) can be reduced to the following :

Every central simple algebra of exponent $n$ is Brauer-equivalent to a tensor product of cross-products, all components of which have the index and exponent equal to $n$.

2) We mention in passing that in some cases of groups of outer types ${ }^{2} \mathrm{~A}_{n},{ }^{2} \mathrm{D}_{n}$, one can prove the Weak Corestriction Principle to hold. This is closely related to the analog of Brauer group for algebras with involution of the second kind first considered by Riehm [Ri2] and then by Parimala and Srinivas [PS] in a more general situation, where they treated also the case of algebras with involution of the first kind. Recently this group has been considered and some related results about this group also have been given in [KMRT] (see also [HKRT]).

3) From the proof of our results it is clear that, in fact, in many cases, the assertion regarding Weak Corestriction Principle (e.g. for finite extension $k^{\prime} / k$ ) proved above holds true if we replace it by stronger statement, that the corestriction (norm)

homomorphism maps any element from the image ("on the $k^{\prime}$-level") into a sum of elements from the image ("on the $k$-level"), (as it was asserted in the original result of Rosset and Tate).

\section{Appendix}

In this section we give some formulas for centers $\tilde{F}$ of simply connected groups $G$, via generators, which are related with Tits index of $G$ (see [Ti]) and they follow 
easily from [St1]. We keep the same notation adopted in [St1].

$B_{n}:$

$$
\tilde{F}=\left\langle h_{n}(-1)\right\rangle
$$

$\mathbf{D}_{\mathbf{n}}, n$ even :

$$
\tilde{F}=\left\langle z_{1}, z_{2}\right\rangle
$$

where

$$
\begin{gathered}
z_{1}=h_{1}(-1) h_{3}(-1) \cdots h_{n-3}(-1) h_{n-1}(-1), \\
z_{2}=h_{1}(-1) h_{3}(-1) \cdots h_{n-3}(-1) h_{n}(-1) .
\end{gathered}
$$

$\mathbf{D}_{\mathbf{n}}, n$ odd :

$$
\tilde{F}=\left\langle h_{1}(-1) h_{3}(-1) \cdots h_{n-2}(-1) h_{n-1}(-i) h_{n}(i)\right\rangle,
$$

where $i=\sqrt{-1}$.

Acknowledgements. This paper was influenced by the works by Rosset and Tate [RT], Merkurjev [Me1 - Me4], Merkurjev and Suslin [MS], Riehm [Ri1]. The work was initiated while the author was staying at Department of Mathematics and Statistics, McMaster University, Hamilton, Ont., Canada, Department of Mathematics, Technion, Israel and the final version was prepared while the author was staying at ICTP, Trieste, Italy, whose support is greatly acknowleged. I would like to thank the referee for very careful reading of the paper and for pointing out some inacuracies in the first version. I would like to thank Professor C. Riehm and Professor J. Tate for their encouragement, and Professor J. -P. Tignol for helpful discussion and for his book.

\section{References}

[Bo] A. Borel, Linear algebraic groups, Second enlarged edition, Graduate Text in Math. v. 126, Springer - Verlag, Berlin - Heidelberg - New York, 1991.

[Bor] M. V. Borovoi, The algebraic fundamental group and abelian Galois cohomology of reductive algebraic groups, Preprint, Max-Plank Inst., MPI/89-90, Bonn, 1990 (another version : Abelian Galois Cohomology of Reductive Groups, Mem. A.M.S. v. 132, No. 626, 1998).

[HKRT] D. Haile, M. -A. Knus, M. Rost and J. -P. Tignol, Algebras of odd degreee with involution, trace form and dihedral extensions, Israel $\mathrm{J}$. Math. 96 (1996), 299-340.

[G] P. Gille, La R-équivalence sur les groupes algébriques réductifs définis sur un corps global, Pub. Math. I. H. E. S. v. 86 (1997), 199-235. 
[KMRT] M. Knus, A. Merkurjev, M. Rost and J. -P. Tignol, The Book of Involutions, A. M. S. Colloquium Pub., v. 44, 1998.

[Me1] A. S. Merkurjev, On the norm residue symbol of degree 2, Sov. Math. Doklady 24 (1981), 546-551.

[Me2] A. S. Merkurjev, A norm principle for algebraic groups, St. Petersburg Math. J. v. 7 (1996), 243-264.

[Me3] A. S. Merkurjev, Milnor K-Theory and Galois Cohomology, Proc. Inter. Cong. Math., Berkley, 1986, v. 1, 389-393.

[Me4] A. S. Merkurjev, Generators and relations for the Brauer group of a field, Proc. Steklov Inst. Math. v. 183 (1990), 163-169.

[MS] A. Merkurjev and A. Suslin, $K$-cohomology of Brauer - Severi varieties and norm residue homomorphism, Math. USSR Izvestya 21 (1983), 307 -340 .

[O] T. Ono, On relative Tamagawa numbers, Ann. Math. 82 (1965), 88 111.

[PS] R. Parimala and V. Srinivas, Analogues of the Brauer group for algebras with involution, Duke Math. J. v. 66 (1992), 207-237.

[Ri1] C. Riehm, The Corestriction of Algebraic Structures, Invent. Math. v. 11 (1970), 73-98.

[Ri2] C. Riehm, The quadratic Schur subgroup over local and global fields, Math. Ann. 283 (1989), 479-489.

[RT] S. Rosset and J. Tate, A reciprocity law for $K_{2}$-traces, Comm. Math. Helv. 58 (1983), 38-47.

[Se] J. -P. Serre, Cohomologie Galoisienne, Lectures Notes in Math., v.5, Springer-Verlag, 1994 (Fifth edition); Galois Cohomology, SpringerVerlag, 1997.

[St1] R. Steinberg,Lectures on Chevalley groups, Yale University Mimeog. Lec. Notes, 1967.

[St2] R. Steinberg, Regular elements of semisimple algebraic groups, Pub. Math. I. H. E. S. v. 25 (1965), 49-80.

[T1] Nguyen Q. Thang, Corestriction principle in non-abelian Galois cohomology, Proc. Japan Acad. v. 74 (1998), 63-67. 
[T2] Nguyen Q. Thang, On corestriction principle in non-abelian Galois cohomology over local and global fields, J. Math. Kyoto Univ. v. 42 (2002), $287-304$.

[Ti] J. Tits, Classification of algebraic semisimple groups, Proc. Sym. Pure Math. A. M. S. v. 9 (1966), 33-62.

This article may be accessed via WWW at http://www.rmi.acnet.ge/hha/ or by anonymous ftp at

ftp://ftp.rmi.acnet.ge/pub/hha/volumes/2003/n1a10/v5n1a10.(dvi,ps,pdf)

Nguyêñ Quôć Thăńg nqthang@thevinh.ncst.ac.vn

Institute of Mathematics

P.O.Box 631 , Bo Ho

Hanoi,

Vietnam 